Mon. Not. R. Astron. Soc. 000,122(2015) Printed 25 August $2021 \quad$ (MN LATEX style file v2.2)

\title{
LAMOST Spectroscopic Survey of the Galactic Anticentre (LSS-GAC): the second release of value-added catalogues
}

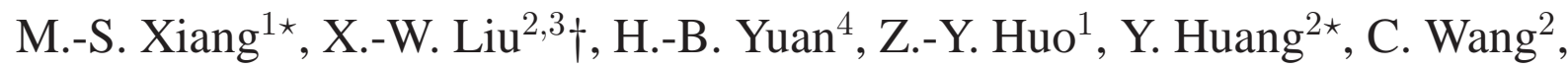 \\ B.-Q. Chen ${ }^{2 \star}$, J.-J. Ren ${ }^{2 \star}$, H.-W. Zhang ${ }^{2}$, Z.-J. Tian ${ }^{2 \star}$, Y. Yang ${ }^{2,4}$, J.-R. Shi ${ }^{1}$, \\ J.-K. Zhao ${ }^{1}$, J. Li ${ }^{5}$, Y.-H. Zhao ${ }^{1}$, X.-Q. Cui ${ }^{6}$, G.-P. Li ${ }^{6}$, Y.-H. Hou ${ }^{6}$, Y. Zhang ${ }^{6}$, \\ W. Zhang ${ }^{1}$, J.-L. Wang ${ }^{1}$, Y.-Z. Wu ${ }^{1}$, Z.-H. Cao ${ }^{1}$, H.-L. Yan ${ }^{1}$, T.-S. Yan ${ }^{1}$, A.-L. Luo ${ }^{1}$, \\ H.-T. Zhang ${ }^{1}$, Z.-R. Bai ${ }^{1}$, H.-L. Yuan ${ }^{1}$, Y.-Q. Dong ${ }^{1}$, Y.-J. Lei ${ }^{1}$, G.-W. Li ${ }^{1}$ \\ 1 Key Laboratory of Optical Astronomy, National Astronomical Observatories, Chinese Academy of Sciences, Beijing 100012, P. R. China \\ 2 Department of Astronomy, Peking University, Beijing 100871, P. R. China \\ 3 Kavli Institute for Astronomy and Astrophysics, Peking University, Beijing 100871, P. R. China \\ 4 Department of Astronomy, Beijing Normal University, Beijing 100875, P. R. China \\ 5 Department of Space Science and Astronomy, Hebei Normal University, Shijiazhuang 050024,China \\ 6 Nanjing Institute of Astronomical Optics \& Technology, National Astronomical Observatories, Chinese Academy of Sciences, Nanjing 210042, P. R. China
}

Received:

\begin{abstract}
We present the second release of value-added catalogues of the LAMOST Spectroscopic Survey of the Galactic Anticentre (LSS-GAC DR2). The catalogues present values of radial velocity $V_{\mathrm{r}}$, atmospheric parameters - effective temperature $T_{\text {eff }}$, surface gravity $\log g$, metallicity $[\mathrm{Fe} / \mathrm{H}], \alpha$-element to iron (metal) abundance ratio $[\alpha / \mathrm{Fe}]([\alpha / \mathrm{M}])$, elemental abundances $[\mathrm{C} / \mathrm{H}]$ and $[\mathrm{N} / \mathrm{H}]$, and absolute magnitudes $\mathrm{M}_{V}$ and $\mathrm{M}_{K_{\mathrm{s}}}$ deduced from 1.8 million spectra of 1.4 million unique stars targeted by the LSS-GAC since September 2011 until June 2014. The catalogues also give values of interstellar reddening, distance and orbital parameters determined with a variety of techniques, as well as proper motions and multi-band photometry from the far-UV to the mid-IR collected from the literature and various surveys. Accuracies of radial velocities reach $5 \mathrm{~km} \mathrm{~s}^{-1}$ for late-type stars, and those of distance estimates range between 10 - 30 per cent, depending on the spectral signal-to-noise ratios. Precisions of $[\mathrm{Fe} / \mathrm{H}]$, $[\mathrm{C} / \mathrm{H}]$ and $[\mathrm{N} / \mathrm{H}]$ estimates reach $0.1 \mathrm{dex}$, and those of $[\alpha / \mathrm{Fe}]$ and $[\alpha / \mathrm{M}]$ reach $0.05 \mathrm{dex}$. The large number of stars, the contiguous sky coverage, the simple yet non-trivial target selection function and the robust estimates of stellar radial velocities and atmospheric parameters, distances and elemental abundances, make the catalogues a valuable data set to study the structure and evolution of the Galaxy, especially the solar-neighbourhood and the outer disk.
\end{abstract}

Key words: Galaxy: abundance - Galaxy: disk - Galaxy: evolution - Galaxy: formation techniques: spectroscopic

\section{INTRODUCTION}

Better understanding the structure, stellar populations, and the chemical and dynamic evolution of the Milky Way is both a challenge and an opportunity of modern galactic astronomy. The Milky Way is the only galaxy whose distribution of stellar populations can be mapped out in full dimensionality — three-dimensional position and velocity, age, as well as photospheric elemental abundances. However, owing to our location inside the Milky Way disk, the

* LAMOST Fellow

† E-mail: x.liu@pku.edu.cn hundreds of billions of Galactic stars are distributed over the whole sky, and our views in the Galactic disk are seriously limited by the interstellar dust extinction. Obtaining the full dimensional distribution of a complete stellar sample is thus a great challenge. It is only recently that comprehensive surveys of Galactic stars become feasible, thanks to the implementation of a number of large-scale photometric and spectroscopic surveys, such as the Sloan Digital Sky Survey (SDSS; York et al. 2000), the Two Micron All Sky Survey (2MASS; Skrutskie et al. 2006), the Apache Point Observatory Galactic Evolution Experiment (APOGEE; Majewski et al. 2010), the LAMOST Experiment for Galactic Understanding and Explo- 
ration (LEGUE; Deng et al. 2012; Zhao et al. 2012) and the Gaia astrometric survey (Perryman et al. 2001).

As a major component of the LEGUE project, the LAMOST Spectroscopic Survey of the Galactic Anticentre (LSS-GAC; Liu et al. 2014; Yuan et al. 2015) is being carried out with the aim to obtain a statistically complete stellar spectroscopic sample in a contiguous sky area around the Galactic anticentre, taking full advantage of the large number of fibers $(4,000)$ and field of view (20 sq.deg.) offered by LAMOST (Cui et al.2012). The survey will allow us to acquire a deeper and more comprehensive understanding of the structure, origin and evolution of the Galactic disk and halo, as well as the transition region between them. The main scientific goals of LSS-GAC include: (a) to characterize the stellar populations, chemical composition, kinematics and structure of the thin and thick disks and their interface with the halo; (b) to understand the temporal and secular evolution of the $\operatorname{disk}(\mathrm{s})$; (c) to probe the gravitational potential and dark matter distribution; (d) to identify star aggregates and substructures in the multi-dimensional phase space; (e) to map the interstellar extinction as a function of distance; (f) to search for and study rare objects (e.g. stars of peculiar chemical composition or hypervelocities); (g) to study variable stars and binaries with multi-epoch spectroscopy.

LSS-GAC plans to collect low-resolution $(R \sim 1800)$ optical spectra $(\lambda \lambda 3700-9000 \AA)$ of more than 3 million stars down to a limiting magnitude of $r \sim 17.8$ mag (to 18.5 mag for selected fields) in a contiguous sky area of over 3400 sq.deg. centred on the Galactic anticentre $\left(|b| \leqslant 30^{\circ}, 150 \leqslant l \leqslant 210^{\circ}\right)$, and deliver spectral classifications, stellar parameters (radial velocity $V_{\mathrm{r}}$, effective temperature $T_{\text {eff }}$, surface gravity $\log g$, metallicity $[\mathrm{Fe} / \mathrm{H}]$, $\alpha$-element to iron abundance ratio $[\alpha / \mathrm{Fe}]$, and individual elemental abundances), as well as values of interstellar extinction and distance of the surveyed stars, so as to build-up an unprecedented, statistically representative multi-dimensional database for the Galactic (disk) studies. The targets of LSS-GAC are selected uniformly in the planes of $(g-r, r)$ and $(r-i, r)$ Hess diagrams and in the (RA, Dec) space with a Monte Carlo method, based on the Xuyi Schmidt Telescope Photometric Survey of the Galactic Anticentre (XSTPSGAC; Zhang et al. 2013, 2014; Liu et al. 2014; Yuan et al. 2015), a CCD imaging photometric survey of $\sim 7000$ sq.deg. with the Xuyi 1.04/1.20m Schmidt Telescope. Stars of all colours are sampled by LSS-GAC. The sampling rates are higher for stars of rare colours, without losing the representation of bulk stars, given the high sampling density ( $\gtrsim 1000$ stars per sq.deg.). This simple yet non-trivial target selection strategy allows for a statistically meaningful study of the underlying stellar populations for a wide range of object class, from white dwarfs (e.g. Rebassa-Mansergas et al. 2015), main sequence turn-off stars (e.g. Xiang et al. 2015b) to red clump giants (e.g. Huang et al. 2015c), after the selection function has been properly taken into account.

As an extension, LSS-GAC also surveys objects in a contiguous area of a few hundred sq.deg. around M31 and M33. The targets include background quasars, planetary nebulae (PNe), H II regions, globular clusters, supergiant stars, as well as foreground Galactic stars. In addition, to make full use of all available observing time, LSS-GAC targets very bright (VB) stars of $r<14$ mag in sky areas accessible to LAMOST $\left(-10^{\circ} \leqslant\right.$ Dec $\left.\leqslant 60^{\circ}\right)$ in poor observing conditions (bright/grey lunar nights, or nights of poor transparency). Those very bright stars comprise an excellent sample supplementary to the main one. Given their relatively low surface densities, at least for the areas outside the disk, LSS-GAC has achieved a very high sampling completeness ( 50 per cent) for those very bright stars, making the sample a golden mine to study the solar-neighbourhood.

Radial velocities and atmospheric parameters (effective temperature $T_{\text {eff }}$, surface gravity $\log g$, metallicity $\left.[\mathrm{Fe} / \mathrm{H}]\right)$ have been deduced from the LSS-GAC spectra for A/F/G/K-type stars using both the official LAMOST Stellar parameter Pipeline (LASP; Wu et al. 2011, 2014) and the LAMOST Stellar Parameter Pipeline at Peking University (LSP3; Xiang et al.2015a). Typical precisions of the results, depending on the spectral signal-to-noise ratio (SNR) and the spectral type, are a few $(5-10) \mathrm{km} \mathrm{s}^{-1}$ for radial velocity $V_{\mathrm{r}}, 100-200 \mathrm{~K}$ for $T_{\text {eff }}, 0.1-0.3$ dex for $\log g, 0.1-0.2$ dex for $[\mathrm{Fe} / \mathrm{H}]$ Xiang et al. 2015a; Luo et al. 2015; Gao et al. 2015; Ren et al. 2016; Wang et al. 2016b). Values of $\alpha$-element to iron abundance ratio $[\alpha / \mathrm{Fe}]$, as well as abundances of individual elements (e.g. $[\mathrm{C} / \mathrm{H}]$ and $[\mathrm{N} / \mathrm{H}]$ ) have also been derived with LSP3 (Li et al. 2016; Xiang et al. 2017), with precisions similar to those achieved by the APOGEE survey for giant stars (Xiang et al. 2017). Efforts have also been made to derive stellar parameters from LAMOST spectra with other pipelines, such as the SSPP (Lee et al. 2015) and the Cannon (Ho et al. 2016). Liu et al. (2015) refine the LASP estimates of $\log g$ using a support vector regression (SVR) model based on Kepler asteroseismic measurements of giant stars. Stellar extinction and distances have been deduced for LSS-GAC sample stars using a variety of methods (Chen et al. 2014; Yuan et al. 2015; Carlin et al. 2015; Wang et al. 2016a), with typical uncertainties of $E_{B-V}$ of about $0.04 \mathrm{mag}$, and of distance between $10-30$ per cent, depending on the stellar spectral type (Yuan et al. 2015).

Following a year-long Pilot Survey, LSS-GAC was initiated in October, 2012, and is expected to complete in the summer of 2017. The LSS-GAC data collected up to the end of the first year of the Regular Survey are public available from two formal official data releases, namely the early (LAMOST EDR; Luo et al. 2012) and first (LAMOST DR1; Luo et al. 2015) data release1. The LAMOST EDR includes spectra and stellar parameters derived with the LASP for stars observed during the Pilot Survey, while the LAMOST DR1 includes stars observed by June, 2013. In addition, there is a public release of LSS-GAC value-added catalogues for stars observed by June, 2013, the LSS-GAC DR 12 (Yuan et al.2015). LSSGAC DR1 presents stellar parameters derived with LSP3, values of interstellar extinction and stellar distance deduced with a variety of methods, as well as magnitudes of broadband photometry compiled from various photometric catalogues (e.g. GALEX, SDSS, XSTPS-GAC, UCAC4, 2MASS and WISE), and values of proper motions from the UCAC4 and PPMXL catalogues and those derived by combing the XSTPS-GAC and 2MASS astrometric measurements, and, finally, stellar orbital parameters (e.g. eccentricity) computed assuming specific Galactic potentials.

This paper presents the second release of value-added catalogues of LSS-GAC (LSS-GAC DR2). LSS-GAC DR2 presents the aforementioned multi-dimensional parameters deduced from $1,796,819$ spectra of 1,408,737 unique stars observed by June, 2014. Compared to LSS-GAC DR1, in addition to a significant increase in stellar number, several improvements to the data reduction and stellar parameter determinations have been implemented, including: (1) An upgraded LAMOST 2D pipeline has been used to process the spectra; (2) The spectral template library used by LSP3 has been updated, adding more than 200 new templates. The

\footnotetext{
1 http://dr1.lamost.org

2 http://lamost973.pku.edu.cn/site/data
} 
atmospheric parameters for all template stars have also been redetermined/calibrated; (3) Values of $\alpha$-element to iron abundance ratio $[\alpha / \mathrm{Fe}]$ have been estimated with LSP3; (4) Accurate values of stellar atmospheric parameters $\left(T_{\text {eff }}, \log g,[\mathrm{Fe} / \mathrm{H}],[\alpha / \mathrm{Fe}]\right)$, absolute magnitudes $\mathrm{M}_{V}$ and $\mathrm{M}_{K_{\mathrm{s}}}$, as well as elemental abundances $[\mathrm{C} / \mathrm{H}]$ and $[\mathrm{N} / \mathrm{H}]$, have also been estimated from the spectra using a multivariate regression method based on kernel-based principal component analysis (KPCA).

The paper is organized as follows. Section 2 describes the observations included in the LSS-GAC DR2, including a brief review of the target selection algorithm and the observational footprint. Section 3 introduces the data reduction briefly. Section 4 presents a detailed description of the improvements in stellar parameter determinations incorporated in LSS-GAC DR2. Section 5 briefly discusses the duplicate observations, which accounts for nearly 30 per cent of all observations. Section 6 introduces the determinations of extinction and distance. Section 7 presents proper motions and derivation of stellar orbital parameters. The format of value-added catalogues is described in Section 8 , followed by a summary in Section 9.

\section{OBSERVATIONS}

To make good use of observing time of different qualities as well as to avoid fibre cross-talking, LSS-GAC stars are targeted by 4 types of survey plates defined in $r$-band magnitude (Liu et al. 2014; Yuan et al. 2015). Stars of $r<14.0$ mag are targeted by very bright (VB) plates, and observed in grey/lunar nights, with typical exposure time of $(2-3) \times 600 \mathrm{~s}$. Stars of $14.0<r \lesssim 16.3 \mathrm{mag}$ are targeted by bright (B) plates, and observed in grey/dark nights, with typical exposure time of $2 \times 1200$ s, whereas stars of $16.3 \lesssim$ $r \lesssim 17.8 \mathrm{mag}$, and of $17.8 \lesssim r<18.5 \mathrm{mag}$ are targeted respectively by medium-bright $(\mathrm{M})$ and faint $(\mathrm{F})$ plates, and observed in dark nights of excellent observing conditions (in term of seeing and transparency), with typical exposure time of $3 \times 1800 \mathrm{~s}$.

By June, 2014, 314 plates $(194 \mathrm{~B}+103 \mathrm{M}+17 \mathrm{~F})$ for the LSS-GAC main survey, 59 plates $(38 \mathrm{~B}+17 \mathrm{M}+4 \mathrm{~F})$ for the M31/M33 survey and 682 plates for the VB survey have been observed. Note that spectra of a few plates could not be successfully processed with the 2D pipeline and are therefore not included in the above statistics. During the survey, some observing time, on the level of one or two grey nights per month has been reserved for monitoring the instrument performance (e.g. throughput and accuracy of fibre positioning; Liu et al. 2014; Yuan et al. 2015). Some of those reserved nights have been used to target some of the LSSGAC plates, yielding another 94 observed plates $(79 \mathrm{~B} / \mathrm{M} / \mathrm{F}+15$ V). Finally, 43 B or M or F LSS-GAC plates were observed from September to October, 2011, when the LAMOST was being commissioned before the start of Pilot Survey. For those 43 plates, the magnitude limits of assigning stars in $\mathrm{B} / \mathrm{M} / \mathrm{F}$ plates are not exactly the same as those adopted during the Pilot and Formal surveys. For convenience, all plates observed using reserved time as well as those collected during the commissioning phase have been grouped into, as appropriately, the LSS-GAC main, M31/M33 and VB survey, respectively, leading to a total of 395 plates for the LSS-GAC main, 100 plates for the M31/M33 and 697 plates of the VB survey, respectively.

The total numbers of spectra collected and unique stars targeted by those plates are listed in Table 1. Table 1 also lists the numbers of spectra and unique stars that are successfully observed, defined by a spectral SNR of higher than 10 either in the blue

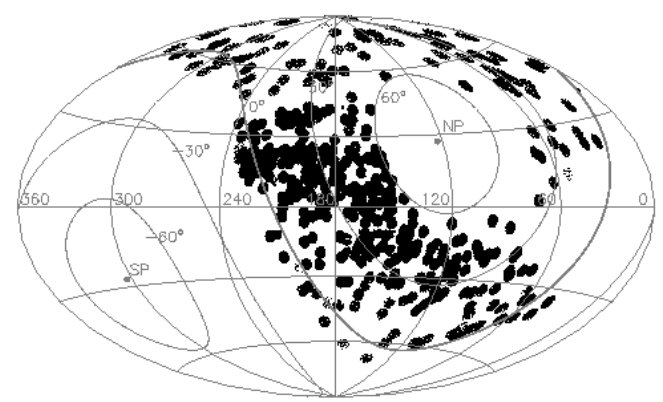

Figure 2. LSS-GAC DR2 footprint for the very bright (VB) plates. To reduce the figure file size, only 1 in 10 observed stars are plotted.

( $\sim 650 \AA)$ or the $(\sim 7450 \AA)$ part of the spectrum (Liu et al. 2014). The numbers of spectra and stars have increased significantly compared to those of LSS-GAC DR1, which contains, for example, 225,522 spectra of 189,042 unique stars of $\operatorname{SNR}(4650 \AA)>10$ for the main survey, and 457,906 spectra of 385,672 unique stars of $\operatorname{SNR}(4650 \AA)>10$ for the VB survey (cf. Yuan et al. 2015). Due to the overlapping of LAMOST fields of view of adjacent plates and the repeating of observations failed to meet the quality control, there is a considerable fraction of stars that have been repeatedly targeted several times. For LSS-GAC DR2, amongst the 948,361 unique stars targeted by the main survey, 71.0, 20.6, 6.0, 1.7, 0.5, 0.1 per cent of the stars are observed by one to six times, respectively. The corresponding fractions for the M31/M33 survey are $60.1,20.4,10.1,4.4,2.5,1.3$ per cent, and those for the VB survey are $73.5,20.5,3.7,1.7,0.3,0.2$ per cent. For those unique stars with $\operatorname{SNR}(4650 \AA)>10$, the corresponding fractions are 83.6, 13.2, 2.4, $0.6,0.1,0.03$ per cent for the LSS-GAC main survey, 72.6, 19.2, $5.6,1.7,0.7,0.2$ per cent for the M31/M33 survey, and 78.3, 17.3, $2.9,1.2,0.2,0.1$ per cent for the VB survey. Note that there are also a small fraction $(\sim 0.8$ per cent $)$ of stars that are targeted by both the LSS-GAC main (or M31/M33) survey and the VB survey. These duplicates were not considered when calculating the above percentage numbers.

Figs. 1 and 2 plot the footprints of stars with either a blue or red spectral SNR higher than 10 for LSS-GAC DR2. The footprints of stars targeted by VB, B and M plates are plotted in separate panels. For the main survey, the strategy is to extend the observations of a stripe of Dec $\sim 29^{\circ}$ to both higher and lower Declinations Yuan et al. 2015). Compared to LSS-GAC DR1, LSS-GAC DR2 has completed two more stripes for $\mathrm{B}$ and $\mathrm{M}$ plates, namely those of Dec $\sim 34^{\circ}$ and $\sim 24^{\circ}$, respectively. A few B and M plates of Galactic latitude $b>35^{\circ}$ were observed using either the reserved time or during the commissioning phase. For the M31/M33 survey, $7 \mathrm{~B}$ and $9 \mathrm{M}$ plates were collected in the second year of the Regular Survey (September 2013 - June 2014), leading to a much larger sky coverage compared to LSS-GAC DR1. Significant progress in the observation of VB plates is seen in LSS-GAC DR2, in term of both the area and continuity of the sky coverage.

Figs. $3-5$ plot the density distributions of stars targeted, as well as those successfully observed (i.e. with a spectral SNR higher than 10 , either in the blue or red), in the colour - magnitude diagrams (CMDs) for the main, M31/M33 and VB surveys, respectively. For the main and M31/M33 surveys, the diagrams are for colour - magnitude combinations $(g-r, r)$ and $(r-i, r)$ used to se- 
Table 1. Numbers of spectra and unique stars (in parentheses) observed by LSS-GAC by June, 2014.

\begin{tabular}{cccc}
\hline & LSS-GAC Main Survey & M31/M33 Survey & VB Survey \\
All SNRs & $1,332,812(948,361)$ & $305,226(171,259)$ & $1,944,525(1,431,219)$ \\
SNR $(4650 \AA)>10$ & $510,531(423,503)$ & $91,921(65,841)$ & $1,194,367(922,935)$ \\
SNR $(7450 \AA)>10$ & $688,459(572,438)$ & $68,380(58,197)$ & $1,358,618(1,050,143)$ \\
SNR $(4650 \AA)>10$ or SNR $(7450)>10$ & $763,723(618,924)$ & $113,597(80,452)$ & $1,397,538(1,075,677)$ \\
\hline \hline
\end{tabular}

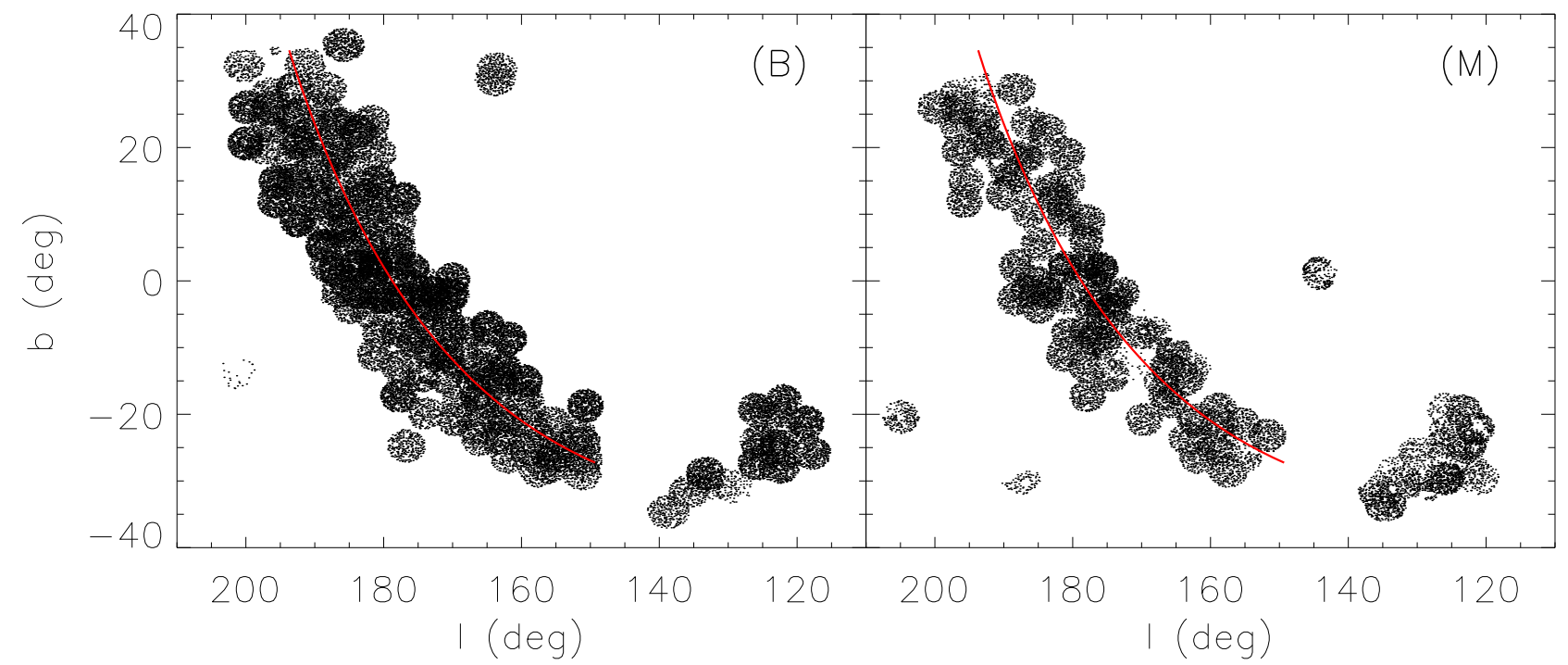

Figure 1. LSS-GAC DR2 footprints in a Galactic coordinate system of stars observed respectively with bright (B; left panel) and medium (M; right panel) plates. The red line denotes a constant Declination of $30^{\circ}$. To reduce the figure file size, only 1 in 10 observed stars are plotted.

lect targets (Yuan et al.2015). Magnitudes of $g, r, i$ bands are from the XSTPS-GAC survey, except for a few plates of high Galactic latitudes, for which magnitudes from the SDSS photometric survey are used. The figures show that, as planned, stars of all colours have been observed. Figs. 3 - 5 also show that the distributions of stars that have either a blue or red spectral SNR higher than 10, as plotted in the middle panels of those three figures, are quite similar to those targeted, plotted in the top panels of the three figures, except for faint ones $(r>17.8 \mathrm{mag})$. Note that for the main and M31/M33 surveys, some stars of either $r>18.5$ or $r<14.0$ mag were observed during the commissioning phase. In contrast, the distributions of stars of $\operatorname{SNR}(4650)>10$ are quite different there are fewer faint stars of red colours. This is caused by a combination of the effects of low intrinsic fluxes in the blue of red stars and the lower throughputs of the spectrographs in the blue compared to those in the red (Cui et al. 2012). For the VB survey, $(g-r, r)$ and $\left(J-K_{\mathrm{s}}, J\right)$ diagrams are plotted. The $g, r, i$ magnitudes are taken from the AAVSO Photometric All-Sky Survey (APASS; Munari et al. 2014), which have a bright limiting magnitude of about 10 mag and a faint limiting magnitude $(10 \sigma)$ of about 16.5 mag in $g, r, i$-bands. For stars of $r>14.0$ mag, magnitudes from the XSTPS-GAC or SDSS surveys are adopted if available. A comparison of stars common to XSTPS-GAC and APASS surveys shows good agreement in both magnitudes and colours for stars of $14.0<r<15.0 \mathrm{mag}$, with differences of just a few $(<5)$ per cent. Due to the heterogeneous input catalogs and magnitude cut criteria used for the VB survey (Yuan et al. 2015), the morphologies of CMD distributions of VB plates are more complicated than those of the main and M31/M33 plates. Nevertheless, due to the high sampling rates of very bright stars (e.g. Xiang et al. 2015b), and the fact that stars of all colours have been observed without a strong colour bias, the selection function can still be well accounted for with some effort and care, if not straightforwardly.

Fig. 6 plots the SNR distribution of spectra for the main, M31/M33 and VB surveys, as well as those of the whole sample for dwarf and giant stars. Only spectra with either SNR(4650A) or $\operatorname{SNR}(7450 \AA)$ higher than 10 are plotted. The number of spectra in logarithmic scale decreases approximately linearly with increasing SNR. SNRs of the red part of the spectra are generally higher than those of the blue part. Also, spectra of the VB survey have generally higher SNRs than those of the main and M31/M33 surveys. Distribution of SNRs for the giants are similar to those of the dwarfs. Here the classification of dwarfs and giants is based on the results of LSP3 (cf. Section 4). For the whole sample, about 36, 57 and 73 per cent of the spectra have a SNR higher than 50, 30 and 20, respectively, in the blue part of the spectrum.

\section{DATA REDUCTION}

The raw spectra of LSS-GAC used to generate the value-added catalogues were processed at Peking University with the LAMOST 2D reduction pipeline (Luo et al. 2012, 2015) to extract the 1D spectra. This process includes several basic reduction steps, including bias subtraction, fibre tracing, fibre flat-fielding, wavelength calibration and sky subtraction. Both fibre tracing and flat-fielding were first carried out using twilight flat-fields. The results were further revised using sky emission lines when processing the object spectra 

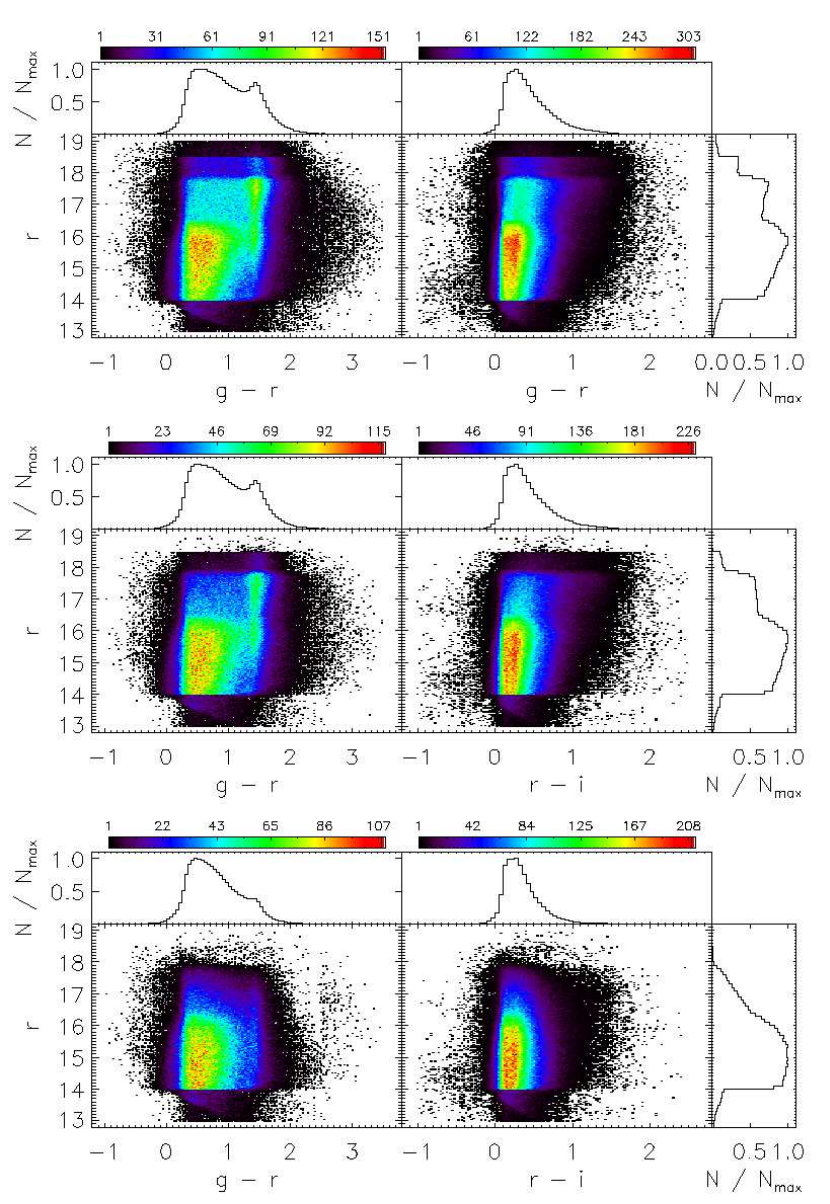

Figure 3. Colour-coded stellar density distributions in the colour-magnitude $(g-r, r)$ and $(r-i, r)$ diagrams for the LSS-GAC main survey. The upper panels show all observed stars, while the middle panels show those with either $\operatorname{SNR}(4650 \AA)>10$ or $\operatorname{SNR}(7450 \AA)>10$, and the lower panels show those with $\operatorname{SNR}(4650 \AA)>10$. The histograms show the one dimensional distributions of stars in colours $(g-r)$ and $(r-i)$ or in magnitude $r$, respectively, normalized to the maximum value.

to account for the potential shifts of fibre traces and the variations in fibre throughput. Typical precision of fibre flat-fielding, as indicated by the dispersions of flat-fields acquired in different days, is better than 1 per cent. Wavelength calibration was carried out using exposures of a Cd-Hg arc lamp for the blue-arm spectra and an Ar-Ne arc lamp for the red-arm spectra. Typically, the residuals of wavelength calibration for the individual arc lines have a mean value close to zero and a standard deviation of $\sim 0.02 \AA$, which corresponds to $\sim 1 \mathrm{~km} \mathrm{~s}^{-1}$ in velocity space. When processing the object spectra, sky emission lines are used to adjust the wavelengths to account for any residual systematic errors in the wavelength calibration and/or potential wavelength drifts between the arc-lamp and object spectra. A comparison of stellar radial velocities with the APOGEE measurements for LAMOST-APOGEE common stars yields an offset of -3 to $-4 \mathrm{~km} \mathrm{~s}^{-1}$ (Xiang et al. 2015c; Luo et al. 2015), and the offset is found to be stable in the past few years, with typical night-to-night variations of about $2 \mathrm{~km} \mathrm{~s}^{-1}$. For each of the 16 spectrographs, about 20 fibres are typically assigned to target sky background for sky subtraction. The numbers of sky fibres allocated for sky measurement are higher for plates of low source surface density, e.g. VB plates of $|b|>10^{\circ}$. To subtract the sky background, the $2 \mathrm{D}$ pipeline creates a super-sky by B-spline fit-

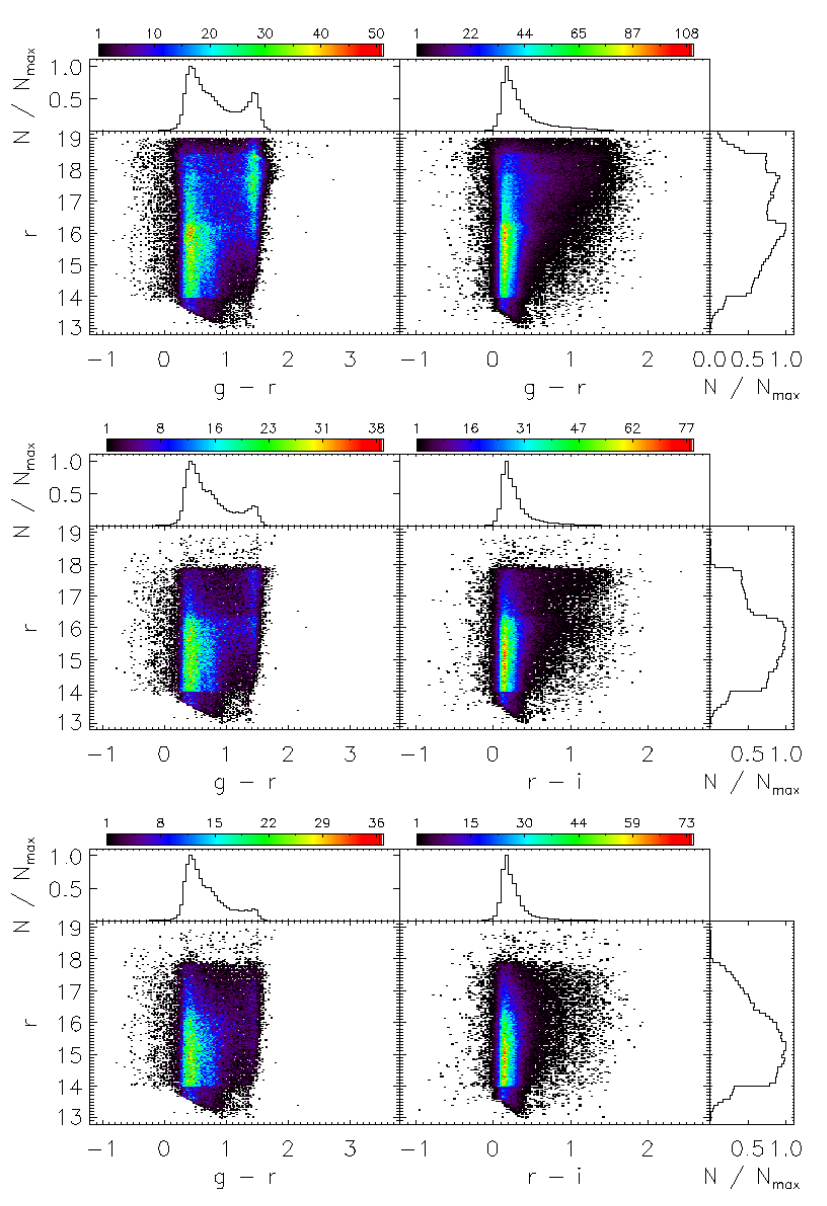

Figure 4. Same as Fig. 3 but for the M31/M33 survey.

ting of fluxes measured by the individual sky fibres. The measured fluxes of sky emission lines in the object spectra are normalized to those of the super-sky spectrum to correct for potential differences of throughputs of fibres used to measure the sky and the objects. The correction assumes that the strengths of the sky emission lines are constant across the sky area covered by a given spectrograph, which is about one square degree, and any differences in sky emission line fluxes as measured by the sky and object fibres, are caused by errors in flat-fielding. The correction also assumes that for the sky background, the continuum scales with emission line flux.

The resultant 1D spectra were then processed with the flux calibration pipeline developed specifically for LSS-GAC to deal with fields of low Galactic latitudes that may suffer from substantial interstellar extinction. The pipeline generates flux-calibrated spectra as well as co-adds the individual exposures of a given plate (Xiang et al. 2015a; Yuan et al. 2015). To deal with the interstellar extinction, the pipeline calibrates the spectra in an iterative way, using F-type stars selected based on the stellar atmospheric parameters yielded by LSP3 as flux standards. The theoretical synthetic spectra from Munari et al. (2005) of the same atmospheric parameters are adopted as the intrinsic spectral energy distributions (SEDs) of the standards. Typical (relative) uncertainties of the spectral response curves (SRCs) thus derived are about 10 per cent for the wavelength range $4000-9000 \AA$ (Xiang et al. 2015c).

Compared to the pipelines used to generate LSS-GAC DR1, a few improvements have been implemented: (1) Several fibres of Spectrograph \#4 are found to be mis-identified before June, 2013 

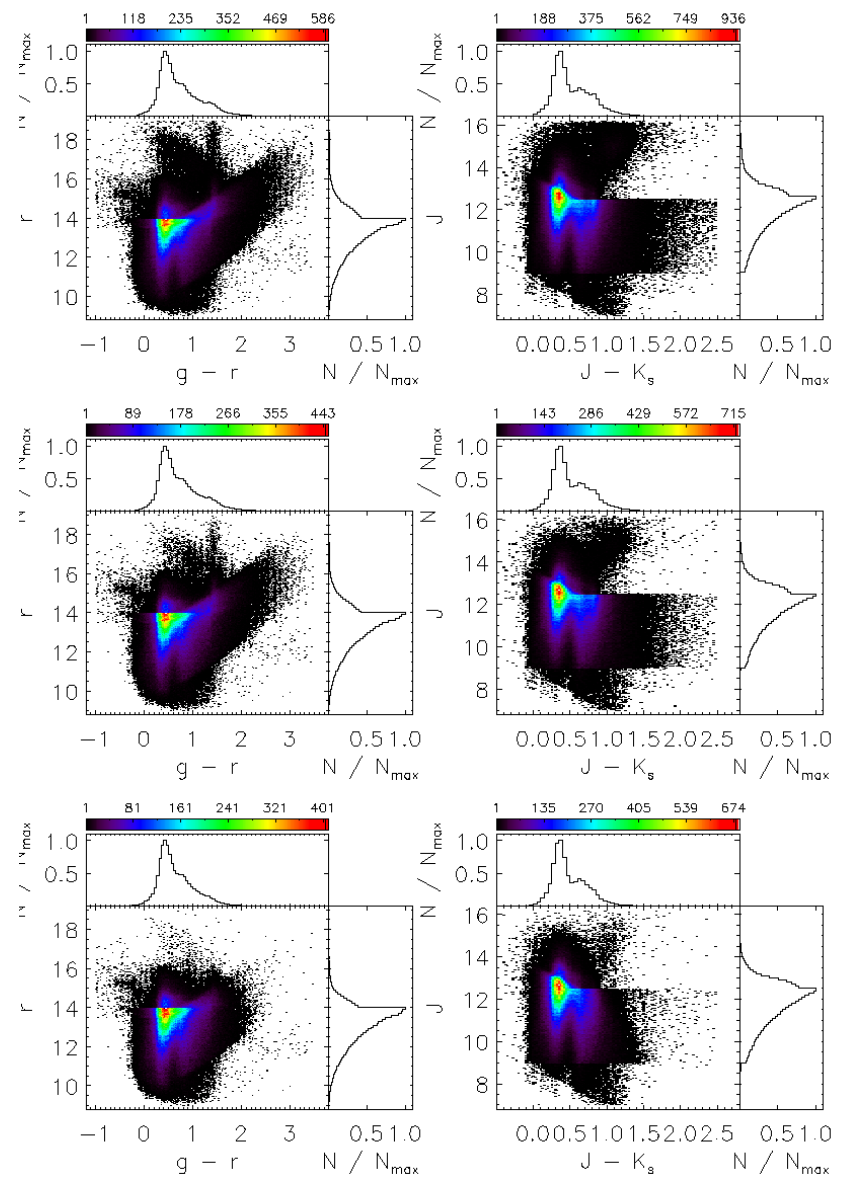

Figure 5. Same as Fig 3 but for the very bright survey. Colour-magnitude diagrams of $(g-r, r)$ and $\left(J-K_{\mathrm{S}}, J\right)$ are shown.

(Luo A.-L., private communication). As a result, the coordinates, magnitudes, spectra and stellar parameters of stars targeted by those fibres were wrongly assigned in LAMOST DR1 (Luo et al. 2015) as well as LSS-GAC DR1 (Yuan et al. 2015). The fibres are: \#76 (87), 87 (79), 79 (95), 95 (84), 84 (76), 44 (31), 31 (46), 46 (26), 26 (44), where the numbers in the brackets are the correct ones. The errors have been corrected; (2) The values of interstellar reddening of flux standard stars are now derived with the star-pair method (Yuan et al. 2015), replacing those deduced by comparing the observed and synthetic colours, as adopted in LSS-GAC DR1. The change is based on the consideration that the star-pair method for extinction determination is model-free, and yields in general more robust results than the method adopted for LSS-GAC DR1 Yuan et al. 2015); (3) For the flux calibration of VB plates, $g, r, i$ magnitudes from the APASS survey (Munari et al. 2014) are combined with 2MASS $J, H, K_{\mathrm{s}}$ magnitudes to derive values of extinction of flux-calibration standard stars. In LSS-GAC DR1, only 2MASS $J, H, K_{\mathrm{s}}$ magnitudes were used. The change should significantly improve the accuracy of extinction estimates for standards used to flux-calibrate VB plates.

Considering that the SNR alone does not give a fully description of the quality of a spectrum, a few flags are now added to image header of a processed spectrum. The first flag is the ratio of (sky-subtracted) stellar flux to the flux of (super-) sky adopted for sky-subtraction. Due to the uncertainties in sky-subtraction, the spectra of some stars, especially those observed under bright lunar
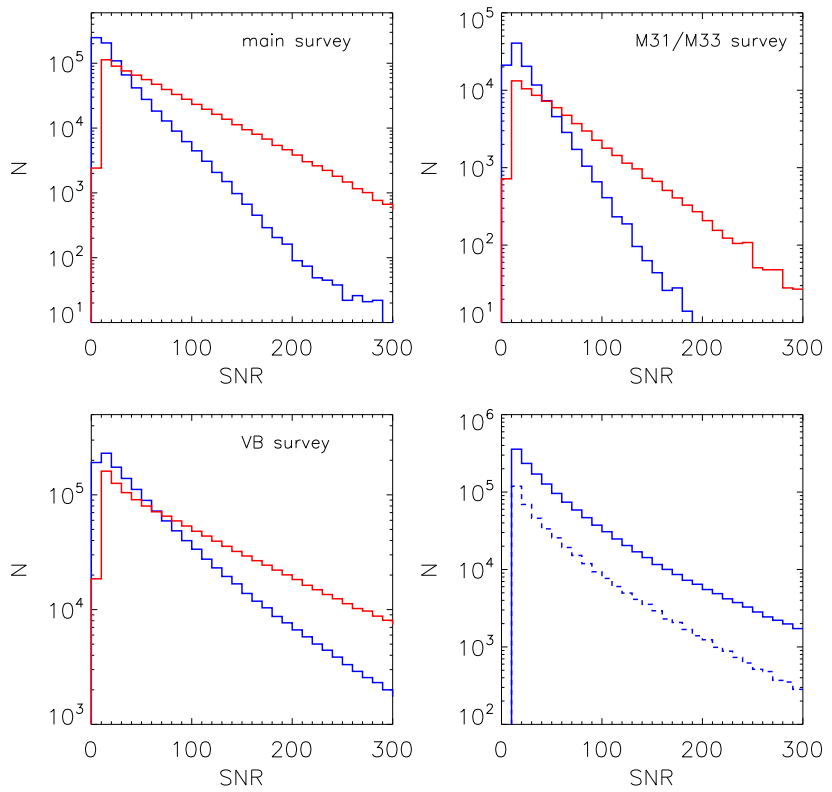

Figure 6. Distribution of spectral SNRs for the main (top left), M31/M33 (top right) and VB (bottom left) surveys. Blue and red lines represent spectral SNR at 4650 and $7450 \AA$, respectively. The bottom right panel plots

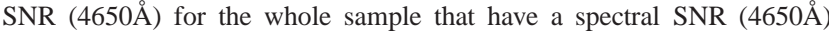
higher than 10 for dwarfs (solid line) and giants (dashed line), respectively.

conditions, may have artificially high SNRs, yet this ratio can be in fact quite small for those spectra. The flag is denoted by 'OBJECT_SKY_RATIO' in the value-added catalogues. A second flag is used to mark fibres that maybe potentially affected by the nearby saturated fibres. Saturation occurs for very bright stars. When a fibre saturates, spectra of nearby fibres, especially those of faint stars, can be seriously contaminated by flux crosstalk, leading to incorrect SNRs and wrongly estimated stellar parameters. When a fibre saturates, stars observed by the adjacent 50 fibers $( \pm 25)$ are now marked by flag 'SATFLAG' in the value-added catalogues. Even when a fiber is not saturated, crosstalk may still occur if the flux of that fibre is very high. To account for such situation, a third flag is introduced. If the spectrum from a given fibre has a SNR higher than 300, then the adjacent $4( \pm 2)$ fibres are assigned a 'BRIGHTFLAG' value of 1 ; otherwise the flag has a value of 0 . For each fibre, the value of maximum SNR of the nearest 5 fibres (the adjacent 4 plus the fibre of concern itself), is also listed as 'BRIGHTSNR' in the value-added catalogues. Finally, a flag has been created to mark bad fibres. Among the 4000 fibres of LAMOST, some have very low throughputs or suffer from serious positioning errors. Spectra yielded by those "bad" fibers cannot be trusted. The number of bad fibres continuously increases with time, and reaches about 200 by June, 2014. Those fibers are marked by 'BADFIBRE' in the value-added catalogues. In addition to those newly created flags, information of observing conditions with regard to the moon (phase, angular distance), the airmass and the pointing position of the telescope are now also included in LSS-GAC DR2. 


\section{STELLAR PARAMETER DETERMINATION: IMPROVEMENTS OF LSP3}

LSS-GAC DR1 presents values of radial velocity $V_{\mathrm{r}}$ and stellar atmospheric parameters (effective temperature $T_{\text {eff }}$, surface gravity $\log g$, metallicity $[\mathrm{Fe} / \mathrm{H}])$ derived from LSS-GAC spectra with LSP3. Since then, a few improvements of LSP3 have been implemented and are included in LSS-GAC DR2, including (1) A number of new spectral templates have been added to the MILES library, and atmospheric parameters of the template stars have been re-determined/calibrated; (2) Several flags are now assigned to describe the best-matching templates that has the characteristics of a, e.g., variable star, binary, double/multiple star or supergiant etc. of a given target spectrum; (3) Values of $\alpha$-element to iron abundance ratio $[\alpha / \mathrm{Fe}]$ have been estimated by template matching with a synthetic spectral library; (4) A multivariate regression method based on kernel-based principal component analysis (KPCA) has been used to obtain an independent set of estimates of stellar atmospheric parameters, including $T_{\text {eff }}, \log g,[\mathrm{M} / \mathrm{H}],[\mathrm{Fe} / \mathrm{H}],[\alpha / \mathrm{M}]$, $[\alpha / \mathrm{Fe}]$, absolute magnitudes $\mathrm{M}_{V}$ and $\mathrm{M}_{K_{\mathrm{S}}}$ as well as individual elemental abundances including $[\mathrm{C} / \mathrm{H}]$ and $[\mathrm{N} / \mathrm{H}]$.

\subsection{Updates to the MILES library}

The original MILES spectral library contains medium-to-low resolution (full-width-at-half-maximum FWHM $\sim 2.5 \AA$ ) long-slit spectra of wavelength range $3525-7410 \AA$ for 985 stars that have robust stellar parameter estimates in the literature, mostly determined with high-resolution spectroscopy (Sánchez-Blázquez et al. 2006; Falcón-Barroso et al. 2011). The MILES library is adopted by LSP3 as templates for estimation of atmospheric parameters from LAMOST spectra. Compared to other template libraries available in the literature, MILES has two advantages. Firstly, the MILES spectra have robust flux calibration and the spectral resolution matches well that of LAMOST spectra. Secondly, the template stars cover a large volume of parameter space $\left(3000<T_{\text {eff }}<\right.$ $40,000 \mathrm{~K}, 0<\log g<5$ dex and $-3.0<[\mathrm{Fe} / \mathrm{H}]<0.5 \operatorname{dex})$.

Still, for the purpose of accurate stellar atmospheric parameter estimation, the MILES library has a few defects in want of improvement. One is the limited spectral wavelength coverage. LAMOST spectra cover the full optical wavelength range of $3700-$ $9000 \AA$, whereas MILES spectra stop at $7410 \AA$ in the red. As a result, LAMOST spectra in the $7400-9000 \AA$ wavelength range have hitherto not been utilized for parameter estimation. There are a few prominent features in this wavelength range that are sensitive abundance indicators, including, e.g. the Ca II $\lambda 8498,8542,8664$ triplet and the $\mathrm{Na}$ I $\lambda 8193,8197$ doublet. In addition, since LSSGAC targets stars of all colours, especially those in the disk, about 30 per cent spectra collected have poor SNRs in the blue but good SNRs in the red. Those stars are either intrinsically red or suffer from heavy interstellar extinction. Stellar parameters for those stars have currently not been derived from LAMOST spectra, by either LSP3 or LASP, due to the fact that both the MILES and ELODIE (adopted by LASP) libraries, do not have wavelength coverage long enough in the red. Another defect of the MILES library is the inhomogenous coverage of stars in the parameter space. As shown in Xiang et al. (2015c), there are holes and clusters in the distribution of MILES stars in the parameter space, leading to some significant artifacts in the resultant parameters. Finally, stellar atmospheric parameters of the original MILES library are collected from various sources in the literature thus suffer from systematic errors. Although Cenarro et al. (2007) have taken effort to homogenize the parameters in order to account for the systematics amongst the values from the different sources, the homogenization was carried out for only a limited temperature range of $4000<T_{\text {eff }}<6300 \mathrm{~K}$. With more data available, there is room of considerable improvement.

To deal with the limited wavelength coverage of the MILES spectra and to improve the coverage and distribution of template stars in the parameter space, an observational campaign is being carried out to observe additional template stars that fill the holes in parameter space, to enlarge the coverage of parameter space, as well as to extend the spectral wavelength coverage to $9200 \AA$, using the NAOC $2.16 \mathrm{~m}$ telescope and the YAO $2.4 \mathrm{~m}$ telescope (Wang et al. in preparation). The project plans to obtain long-slit spectra covering 3600 - 9200A for some 900 template stars newly selected from the PASTEL catalog (Soubiran et al. 2010), a compilation of stars with robust stellar parameters, mostly inferred from high resolution spectroscopy. The project will also extend the wavelength coverage of all the original MILES spectra to $9200 \AA$. In the current work, 267 new template spectra obtained by the campaign by October, 2015 have been added to the MILES library to generate parameter estimates presented in LSS-GAC DR2. In addition, to reduce the systematic and random errors of the atmospheric parameters of the template stars, Huang et al. (in preparation) have re-calibrated/determined the atmospheric parameters of all template stars, both old and newly selected. In doing so, the recent determinations of parameters of template stars available from the PASTEL catalogues have been adopted, replacing the older values used by the original MILES library. The updated values of metallicity are then used to calculate effective temperatures using the newly published metallicity-dependent colour-temperature relations of Huang et al. (2015a), deduced from more than two hundred nearby stars with direct, interferometric angular size measurements and Hipparcos parallaxes. Values of surface gravity are re-determined using Hipparcos parallaxes (Perryman et al. 1997; Anderson \& Francis 2012) and stellar isochrones from the Dartmouth Stellar Evolution Database (Dotter et al. 2008). Values of $[\mathrm{Fe} / \mathrm{H}]$ are then re-calibrated to the standard scale of Gaia-ESO survey (Jofré et al.2015). Fig. 6 plots the newly calibrated/determined parameters of the template stars in the $T_{\text {eff }}-\log g$ and $T_{\text {eff }}-[\mathrm{Fe} / \mathrm{H}]$ planes. Note that in generating LSS-GAC DR1, 85 of the original MILES stars were abandoned because there were no complete parameter estimates in the literatures. Those stars are now included in the library as their parameters have been re-determined. Also, with the newly selected and observed templates added to the library, we have now discarded the 400 interpolated spectra used when generating LSS-GAC DR1.

\section{2 $[\alpha / \mathrm{Fe}]$ estimation by matching with synthetic spectra}

$\alpha$-element to iron abundance ratio $[\alpha / \mathrm{Fe}]$ is a good indicator of the Galactic chemical enrichment history (e.g. Lee et al.2011), and thus valuable to derive. To estimate ratios $[\alpha / \mathrm{Fe}]$ from LAMOST spectra, a method of template matching based on $\chi^{2}$-fitting with synthetic spectra is developed for LSP3. Details about the method and robustness tests of the deduced $[\alpha / \mathrm{Fe}]$ values are described in Li et al. (2016). Here we briefly summarize the method and point out a few improvements that may lead to better results.

The synthetic library used to estimate $[\alpha / \mathrm{Fe}]$ was generated with the SPECTRUM code (Grav 1999) of version 2.76, utilizing the Kurucz stellar model atmospheres of Castelli \& Kurucz (2004). The solar $[\alpha / \mathrm{Fe}]$ ratio is set to zero, and the $\alpha$-enhanced grids are generated by scaling the elemental abundances of $\mathrm{O}, \mathrm{Mg}, \mathrm{Si}, \mathrm{S}$, 


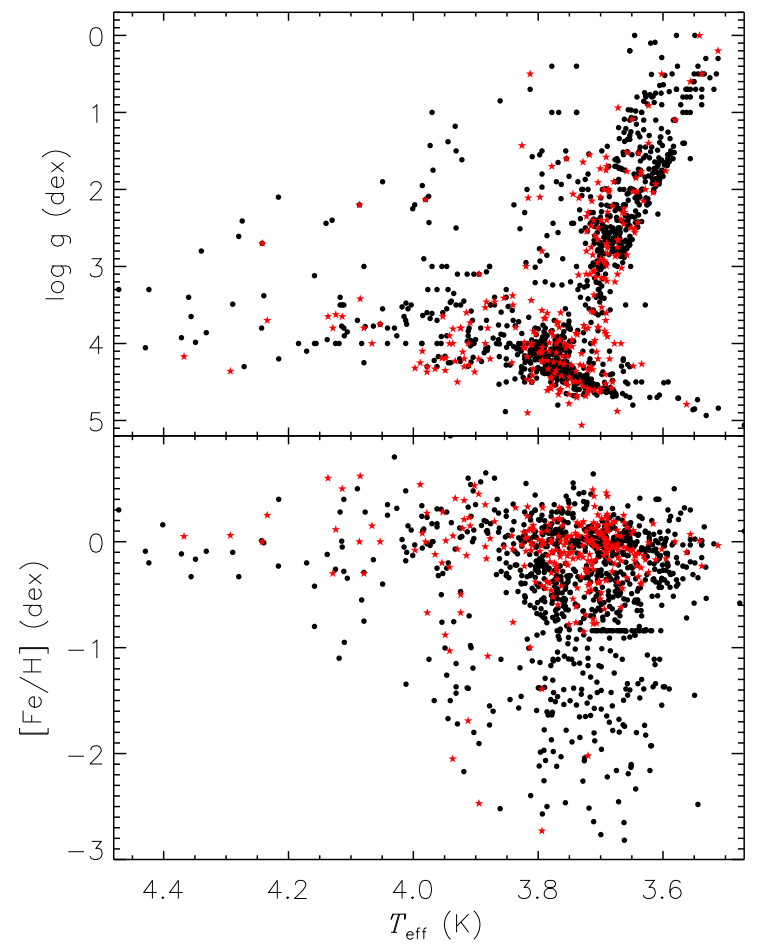

Figure 7. Distributions of template stars used by LSP 3 in the $T_{\text {eff }}-\log g$ and $T_{\text {eff }}-[\mathrm{Fe} / \mathrm{H}]$ planes. Black dots represent stars in the original MILES library, while red dots represent 267 newly observed templates employed in the generation of LSS-GAC DR2 (see the text).

Table 2. Grids of KURUCZ synthetic spectra for $[\alpha / \mathrm{Fe}]$ estimation.

\begin{tabular}{ccc}
\hline Parameter & Range & Step \\
\hline$T_{\text {eff }}$ & {$[4000,8000] \mathrm{K}$} & $100 \mathrm{~K}$ \\
$\log g$ & {$[0.0,5.0] \mathrm{dex}$} & $0.25 \mathrm{dex}$ \\
{$[\mathrm{Fe} / \mathrm{H}]$} & {$[-4.0,0.5] \mathrm{dex}$} & 0.2 dex for $[\mathrm{Fe} / \mathrm{H}]<-1.0 \mathrm{dex}$ \\
& $0.1 \mathrm{dex}$ for $[\mathrm{Fe} / \mathrm{H}]>-1.0 \mathrm{dex}$ \\
{$[\alpha / \mathrm{Fe}]$} & {$[-0.4,1.0] \mathrm{dex}$} & $0.1 \mathrm{dex}$ \\
\hline
\end{tabular}

$\mathrm{Ca}$ and $\mathrm{Ti}$, and those of $\mathrm{C}$ and $\mathrm{N}$ abundances are also enhanced by the amount of $\alpha$-elements. Lines from more then 15 diatomic molecule species, including $\mathrm{H}_{2}, \mathrm{CH}, \mathrm{NH}, \mathrm{OH}, \mathrm{MgH}, \mathrm{AlH}, \mathrm{SiH}$, $\mathrm{CaH}, \mathrm{C}_{2}, \mathrm{CN}, \mathrm{CO}, \mathrm{AlO}, \mathrm{SiO}, \mathrm{TiO}$ and $\mathrm{ZrO}$, are taken into account in the calculation of the opacity. Isotope lines are also taken into account. In total, 320,000 synthetic spectra are generated. Table 2 lists the parameter ranges and steps of the grids. Note that the grids adopted here have a step of $0.1 \mathrm{dex}$ in $[\alpha / \mathrm{Fe}]$, half of the value used in Li et al. (2016). All the computed synthetic spectra have a resolution of $2.5 \AA$ FWHM, and is invariant with wavelength.

For a target spectrum with atmospheric parameters $T_{\text {eff }}, \log g$ and $[\mathrm{Fe} / \mathrm{H}]$ yielded by LSP3, the synthetic spectra are interpolated to generate a set of spectra that have the same atmospheric parameters as the target for all grid values of $[\alpha / \mathrm{Fe}]$. Values of $\chi^{2}$ between the target and the individual interpolated synthetic spectra are then calculated. A Gaussian plus a second order polynomial is then used to fit the deduced $\chi^{2}$ as a function of $[\alpha / \mathrm{Fe}]$. The value of $[\alpha / \mathrm{Fe}]$ that yields minimum $\chi^{2}$ is taken to be the $[\alpha / \mathrm{Fe}]$ ratio of the target spectrum. To compute $\chi^{2}$, Li et al. (2016) use spectral segments $4400-4600 \AA$ and $5000-5300 \AA$. The $4400-4600 \AA$ seg- ment contains mainly Ti features, while that of $5000-5300 \AA$ contains mainly $\mathrm{Mg}$ I features, as well as a few features of $\mathrm{Ca}$, Ti and $\mathrm{Si}$. Note that given the low resolution as well as limited SNRs of LAMOST spectra, Ca, Ti and Si features within those two spectral segments contribute in fact only a small fraction of the calculated values of $\chi^{2}$, and are therefore not very useful for the determination of $[\alpha / \mathrm{Fe}]$. In metal-rich stars, the $\mathrm{Mg} \mathrm{I} b$ features are in general prominent enough for a robust determination of $[\alpha / \mathrm{Fe}]$. However, in metal-poor $([\mathrm{Fe} / \mathrm{H}]<-1.0 \mathrm{dex})$ stars, the $\mathrm{Mg}$ I b lines become less prominent so that the $[\alpha / \mathrm{Fe}]$ have larger uncertainties. In the current work, in order to improve precision of $[\alpha / \mathrm{Fe}]$ estimates, especially for metal-poor stars, we have opted to include the 3910 - $3980 \AA$ spectral segment that contains the Ca II HK lines in the calculation of $\chi^{2}$ values. Meanwhile, as an option, we also provide results yielded using the exactly same spectral segments as Li et al. (2016). This is useful considering that the strong Ca II HK lines in model spectra for metal-rich stars maybe not accurately synthesized.

The resolution of LAMOST spectra from individual fibers varies from one to another, as well as with wavelength Xiang et al. 2015c). To account for this in template matching, the synthetic spectra are degraded in resolution to match that of the target spectrum. The latter is derived utilizing the arc spectrum. The deduced resolution as a function of wavelength is further scaled to match the resolution yielded by sky emission lines detected in the target spectrum in order to account for systematic variations of spectral resolution between the arc and target exposures. Typically, for a given spectrograph, fibre to fibre variations of spectral resolution amount to $0.3 \AA$, rising to $0.5-1.0 \AA$ among the different spectrographs. Systematic variations of spectral resolution between the arc and target exposures are found to be typically $0.2 \AA$.

In addition, in order to allow for possible uncertainties in the input atmospheric parameters $T_{\text {eff }}, \log g$ and $[\mathrm{Fe} / \mathrm{H}]$ yielded by LSP3 as well as any possible mismatch between the LSP3 atmospheric parameters and those of the Kurucz stellar model atmospheres, in the current work, we have opted not to fix the input values of $T_{\text {eff }}, \log g$ and $[\mathrm{Fe} / \mathrm{H}]$ as yielded by LSP3, but allow them to vary in limited ranges around the initial values. The ranges are set to $2 \sigma$ uncertainties of the parameters concerned, with lower limits of $500 \mathrm{~K}, 0.5 \mathrm{dex}$ and $0.5 \mathrm{dex}$ and upper limits of $1000 \mathrm{~K}, 1.0 \mathrm{dex}$ and $1.0 \mathrm{dex}$ for $T_{\mathrm{eff}}, \log g$ and $[\mathrm{Fe} / \mathrm{H}]$, respectively. For each grid value of $[\alpha / \mathrm{Fe}]$, the synthetic spectrum that has atmospheric parameters $T_{\text {eff }}, \log g$ and $[\mathrm{Fe} / \mathrm{H}]$ within the above ranges and fits the target spectrum best (i.e. yielding the smallest $\chi^{2}$ ) is taken as the choice of synthetic spectrum when fitting and deriving $[\alpha / \mathrm{Fe}]$ using the technique described in Li et al. 2016.

Values of $[\alpha / \mathrm{Fe}]$ are derived with the above algorithm for all LSS-GAC DR2 stars of a spectral SNR higher than 15. The left panel in the third row of Fig. 9 plots the density distribution of LSS-GAC DR2 dwarf stars in the $[\mathrm{Fe} / \mathrm{H}]-[\alpha / \mathrm{Fe}]$ plane. Here the $[\alpha / \mathrm{Fe}]$ are those derived using spectra including the $3910-$ $3980 \AA$ segments. The figure shows an $[\alpha / \mathrm{Fe}]$ plateau for metalpoor $([\mathrm{Fe} / \mathrm{H}]<-1.0 \mathrm{dex})$ stars, at a median value about $0.4 \mathrm{dex}$. For more metal-rich stars, $[\alpha / \mathrm{Fe}]$ decreases with increasing $[\mathrm{Fe} / \mathrm{H}]$, reaching a median value of zero near the solar metallicity, which means that the zero-point offset, i.e. deviations of $[\alpha / \mathrm{Fe}]$ values from zero at solar metallicity, is small, which is in contrast to Li et al. (2016) who find a zero-point offset of -0.12 dex. However, we indeed find a zero-point offset of about -0.1 dex for $[\alpha / \mathrm{Fe}]$ derived using only the $4400-4600 \AA$ and $5000-5300 \AA$ segments, which is basically consistent with Li et al. (2016). The offset is found to be mainly contributed by the $4400-4600 \AA$ segment. 
The causes of this difference are not fully understand yet. We suspect there may be some unrealistic inputs in either the atmosphere model or the atomic and molecular data used to generate the synthetic spectra. Note that here we opt not to introduce any external corrections on the estimated $[\alpha / \mathrm{Fe}]$. Random errors of $[\alpha / \mathrm{Fe}]$ induced by spectral noises, as estimated by comparing the results deduced from duplicate observations, are a function of spectral SNR and atmospheric parameters $T_{\text {eff }}, \log g$ and $[\mathrm{Fe} / \mathrm{H}]$, and have typical values that decrease from $\sim 0.1$ to $\sim 0.05 \mathrm{dex}$ as the spectral SNR increases from 20 to a value higher than 50 . To provide a realistic error estimate for $[\alpha / \mathrm{Fe}]$, the random error induced by spectral noises is combined with the method error, which is assumed to have a constant value of $0.09 \mathrm{dex}$, estimated by a comparison with highresolution measurements (cf. Li et al. 2016).

For giant stars, $[\alpha / \mathrm{Fe}]$ estimated with the above algorithm exhibits a zero-point offsets between 0.1 and 0.2 dex, probably caused by inadequacies of the synthetic spectra for giant stars. In this work, no corrections for those offsets are applied. Note that for giant stars, $[\alpha / \mathrm{Fe}]$ values are also estimated with the KPCA regression method using the LAMOST-APOGEE common stars as the training data set (cf.§4.3), and the resultant values are presented as the recommended ones (cf.§4.6).

\subsection{Stellar atmospheric parameters estimated with KPCA method}

The LSP3 version used to generate LSS-GAC DR1 estimates stellar atmospheric parameters $T_{\text {eff }}, \log g$ and $[\mathrm{Fe} / \mathrm{H}]$ with a $\chi^{2}$-based weighted-mean algorithm. The algorithm achieves a high precision in the sense that random errors of the deduced parameters induced by spectral noises are well controlled, even for stars of SNRs as low as 10 . Nevertheless, parameters estimated with the weighted-mean algorithm suffer from several artifacts. One is the so-called 'suppression effect' - values of the derived $\log g$ are narrowed down to an artificially small range. This is partly caused by the fact that $\chi^{2}$ calculated from a LAMOST spectrum with respect to a template spectrum is only moderately sensitive to $\log g$, thus the sets of templates used to calculate the weighted-mean values of $\log g$ for the individual target sources often have similar distributions in $\log g$. Another artifact is the so-called 'boundary effect' - parameters of stars with true parameters that pass or are close to the boundary of parameter space covered by the templates are often either underestimated or overestimated systematically by the weighted-mean algorithm. This effect is especially serious for $[\mathrm{Fe} / \mathrm{H}]$ and $\log g$ estimation. Finally, due to the inhomogeneous distribution of templates in the parameter space, moderate clustering effect is also seen in the deduced parameter values.

To overcome the above defects of the weighted-mean algorithm, a regression method based on Kernel-based Principal Component Analysis (KPCA) has recently been incorporated into LSP3 (Xiang et al.2017). The method is implemented in a machine learning scheme. A training data set is first defined to extract non-linear principal components (and the loading vectors) as well as to buildup regression relations between the principal components and the target parameters. Four training data sets are defined for the determination of specific sets of parameters of specific types of stars. They are: (1) The MILES library for the estimation of $T_{\text {eff }}$ and $[\mathrm{Fe} / \mathrm{H}]$ of $\mathrm{A} / \mathrm{F} / \mathrm{G} / \mathrm{K}$ stars, and for the estimation of $\log g$ of stars that have $\log g$ values larger than 3.0 dex as given by the weighted-mean algorithm. The latter stars are mainly dwarfs and sub-giants; (2) A sample of LAMOST-Hipparcos common stars with accurate parallax (thus distance and absolute magnitude) measurements for the estimation of absolute magnitudes $\left(\mathrm{M}_{V}\right.$ and $\left.\mathrm{M}_{K_{\mathrm{s}}}\right)$ directly from LAMOST spectra; (3) A sample of LAMOST-Kepler common stars with accurate asteroseismic $\log g$ measurements for the estimation of $\log g$ of giant stars; and (4) A sample of LAMOSTAPOGEE common stars for the estimation of metal abundance $[\mathrm{M} / \mathrm{H}], \alpha$-element to iron (metal) abundance ratio $[\alpha / \mathrm{Fe}]([\alpha / \mathrm{M}])$, and of individual elemental abundances including $[\mathrm{Fe} / \mathrm{H}],[\mathrm{C} / \mathrm{H}]$ and $[\mathrm{N} / \mathrm{H}]$ for giant stars. A detailed description of the implementation and test of the method is presented in Xiang et al. (2017). Here we note two updates of the implementation with respect to those of Xiang et al. (2017). One is that a unified number of principal components (PCs) of 100 is adopted except for the estimation of absolute magnitudes with the LAMOST-Hipparcos training set, for which both 100 and 300 PCs are adopted. Another modification is that additional training stars have been added to the LAMOSTKepler and LAMOST-APOGEE training sets as a result that more common stars become available as the surveys progress. Both training sets now contain (exactly) 3000 stars.

Compared to the weighted-mean results, $\log g$ and $[\mathrm{Fe} / \mathrm{H}]$ derived with the KPCA regression method using the MILES training set have been found to suffer from less systematics, and thus better for statistical analyses. A comparison with asteroseismic measurements shows that for stars of $T_{\text {eff }}<6000 \mathrm{~K}$ and $[\mathrm{Fe} / \mathrm{H}]>$ -1.5 dex, uncertainties of KPCA $\log g$ estimates can be as small as 0.1 dex for spectral SNRs higher than 50. The uncertainties increase substantially as the SNR deteriorates, reaching $\sim 0.2$ dex at a SNR of 20 (Xiang et al. 2017). Similarly, comparisons with [Fe/H] determinations from high resolution spectroscopy show that uncertainties of $\mathrm{KPCA}[\mathrm{Fe} / \mathrm{H}]$ estimates are $\sim 0.1 \mathrm{dex}$ for good SNRs. For metal-poor $([\mathrm{Fe} / \mathrm{H}]<-1.5 \mathrm{dex})$ or hot $\left(T_{\text {eff }}>8000 \mathrm{~K}\right)$ stars, KPCA estimates of both $\log g$ and $[\mathrm{Fe} / \mathrm{H}]$ become less reliable, and should be used with caution. Systematic differences between KPCA and weighted-mean estimates of $T_{\text {eff }}$ exist for stars hotter than $\sim 6500 \mathrm{~K}$, with deviations reach as much as $\sim 300 \mathrm{~K}$ for stars of $T_{\text {eff }} \sim 7500 \mathrm{~K}$. More studies are needed to understand the cause of deviations. For the time being, we recommend weighted-mean $T_{\text {eff }}$ values as they are estimated in a straightforward way and well validated (Xiang et al. 2015c). However, note that weighted-mean estimates of $T_{\text {eff }}$ suffer from clustering effect so that they may weakly clump in the parameter space on the scale of a few tens Kelvin, while the KPCA estimates of $T_{\text {eff }}$ do not have such problem.

Surface gravities of giant stars of $T_{\text {eff }}<5600$ and $\log g<$ 3.8 dex are estimated with the KPCA method using the LAMOSTKepler training set. Here the cuts of $T_{\text {eff }}$ and $\log g$ are based on values yielded by the weighted-mean method. The KPCA estimate of $\log g$ is likely to be accurate to 0.1 dex given a spectral SNR higher than 50 . The uncertainty increases to $\sim 0.2$ dex at a SNR of 20 (Huang et al. 2015b; Xiang et al. 2017).

Absolute magnitudes $\mathrm{M}_{V}$ and $\mathrm{M}_{K_{\mathrm{s}}}$ of all stars with a weighted-mean effective temperature lower than $12,000 \mathrm{~K}$ are estimated directly from LAMOST spectra using the LAMOSTHipparcos training set. Two sets of absolute magnitudes are given, corresponding to respectively 100 and 300 PCs adopted for parameter estimation. For absolute magnitudes estimated using $100 \mathrm{PCs}$, typical uncertainties are 0.3-0.4 mag in both $\mathrm{M}_{V}$ and $\mathrm{M}_{K_{\mathrm{s}}}$ for a spectral SNR higher than 50, and the values increase to $\sim 0.6 \mathrm{mag}$ at a SNR of 20. For absolute magnitudes estimated using $300 \mathrm{PCs}$, uncertainties are only $0.2-0.3 \mathrm{mag}$ for a spectral SNR higher than 50 , but the results are more sensitive to SNR, with a typical uncertainty of $\sim 0.7 \mathrm{mag}$ at a SNR of 20 .

A significant advantage of estimating absolute magnitudes di- 

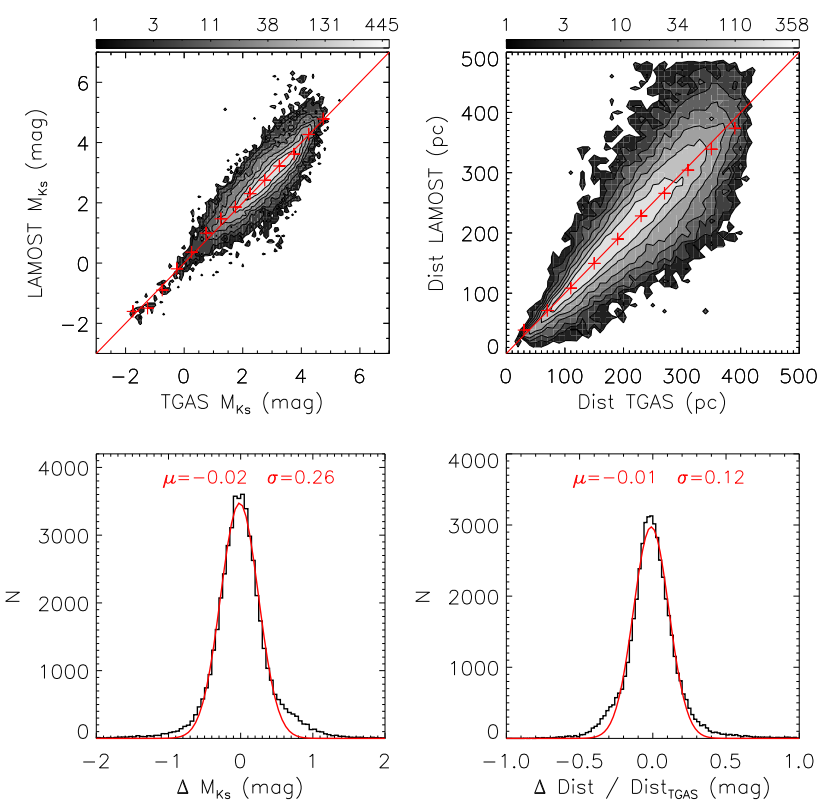

Figure 8. Comparison of absolute magnitudes and distances with those inferred from Gaia TGAS parallax for 50,000 LAMOST-TGAS common stars that have a TGAS-based magnitude error smaller than 0.2 mag. The upper panels shows colour-coded contours of stellar number density in logarithmic scale. Crosses in red are median values of our estimates calculated in bins of the TGAS-based values. The lower panels plot distribution of differences of magnitudes and distances between our estimates and the TGASbased values. Red lines are Gaussian fits to the distribution, with the mean and dispersion of the Gaussian marked in the plot.

rectly from the observed spectra is that the magnitudes as well as the resultant distance moduli, are model independent. Fig. 8 plots a comparison of the estimates of $\mathrm{M}_{K_{\mathrm{s}}}$ for $300 \mathrm{PCs}$ as well as the distances thus estimated with results inferred from the Gaia TGAS parallaxes (Lindegren et al. 2016) for a sample of 50,000 LAMOST-TGAS common stars that have a TGAS-based magnitude error smaller than 0.2 mag. Here the TGAS distances and $\mathrm{M}_{K_{\mathrm{s}}}$ values are derived using values of interstellar extinction derived with the star pair method (cf. Section 6.1). The figure shows very good agreement between our estimates and the TGAS results. Systematics in both $\mathrm{M}_{K_{\mathrm{s}}}$ and distance estimates are negligible, and the dispersion is only 0.26 mag for $\mathrm{M}_{K_{\mathrm{s}}}, 12$ per cent for distance. A similar comparison of results yielded using 100 PCs yields a mean difference of $0.04 \mathrm{mag}$ and a dispersion of $0.29 \mathrm{mag}$ in $\mathrm{M}_{K_{\mathrm{s}}}$, and a mean difference of -2 per cent and a dispersion of 13 per cent in distance estimates. Of course, spectral SNRs for the LAMOSTTGAS common stars are very high, which have a median value of 150, as the stars are very bright. Note that Fig. 8 shows also a positive non-Gaussian tail in the difference of $\mathrm{M}_{K_{\mathrm{s}}}$, with a corresponding negative tail in the difference of distance. This non-Gaussian tail is likely caused by binary stars, for which the estimates of absolute magnitudes from LAMOST spectra are only marginally affected, whereas the photometric magnitudes are underestimated respect to those assuming single stars. Note that not all the LAMOSTTGAS stars used for the comparison can be found in the LSS-GAC value-added catalogues as many of them are targeted by other survey projects of LAMOST. The spectra of those stars have been processed with LSP3 and the data are only internally available for the moment.
Metal (iron) abundance $[\mathrm{M} / \mathrm{H}]([\mathrm{Fe} / \mathrm{H}]), \alpha$-element to metal (iron) abundance ratio $[\alpha / \mathrm{M}]([\alpha / \mathrm{Fe}])$, as well as abundances of carbon and nitrogen, $[\mathrm{C} / \mathrm{H}]$ and $[\mathrm{N} / \mathrm{H}]$, are estimated using the LAMOST-APOGEE training set. Xiang et al. (2017) have demonstrated that the results have a precision comparable to those deduced from APOGEE spectra with the ASPCAP pipeline García Pérez et al. 2015; Holtzman et al. 2015). Specifically, estimates of $[\mathrm{M} / \mathrm{H}],[\mathrm{Fe} / \mathrm{H}],[\mathrm{C} / \mathrm{H}]$ and $[\mathrm{N} / \mathrm{H}]$ have a precision better than 0.1 dex given a spectral SNR high than 30 , and $\sim 0.15 \mathrm{dex}$ for a SNR of 20. We note however, since the APOGEE stellar parameters are not externally calibrated except for $[\mathrm{M} / \mathrm{H}]$, any systematics in the APOGEE results propagated into ours through the training set. As pointed out by Holtzman et al. (2015), APOGEE estimates of elemental abundances may suffer from systematic biases of about $0.1-0.2$ dex. For $[\mathrm{M} / \mathrm{H}]$, the APOGEE values are calibrated to $[\mathrm{Fe} / \mathrm{H}]$ measurements of star clusters for $[\mathrm{Fe} / \mathrm{H}]$ range $[-2.5,0.5]$ dex. Nevertheless, it is found that $[\mathrm{Fe} / \mathrm{H}]([\mathrm{M} / \mathrm{H}])$ estimated with the LAMOST-APOGEE training set are 0.1 dex systematically higher than those estimated with the MILES library. Such an overestimation is also confirmed by examining common stars with high resolution spectroscopic $[\mathrm{Fe} / \mathrm{H}]$ measurements available from the PASTEL catalogue. The discrepancy is likely due to an offset in absolute value between the APOGEE and MILES (PASTEL) metallicities. The estimated $[\alpha / \mathrm{M}]([\alpha / \mathrm{Fe}])$ values have a typical precision of $0.03-0.06$ dex given a spectral SNR higher than 20. Xiang et al. (2017) have demonstrated that, as a consequence of such a high precision, a clear distinction between the sequences of thick and thin disk stars is seen in the $[\mathrm{M} / \mathrm{H}]([\mathrm{Fe} / \mathrm{H}])-[\alpha / \mathrm{M}]$ ( $[\alpha / \mathrm{Fe}])$ plane, quite similar to that revealed by results from highresolution spectroscopy.Note however that, for stars with $[\alpha / \mathrm{M}]$ higher than 0.30 dex or lower than 0.0 dex, the KPCA values are probably systematically underestimated or overestimated due to a lack of training stars of such abundance ratios. In addition, given that APOGEE $[\alpha / \mathrm{M}]([\alpha / \mathrm{Fe}])$ values are not externally calibrated, there may also be some systematics hided in our results.

\subsection{Estimation of parameter errors}

Errors of the deduced stellar parameters are estimated in a statistical way. Parameter errors contributed by both spectral noises and inadequacies of the method are taken into account. For $T_{\text {eff }}, \log g$ and $[\mathrm{Fe} / \mathrm{H}]$ estimated by the weighted-mean algorithm, or those by the KPCA method using the MILES training set, as well as $\mathrm{M}_{V}$ and $\mathrm{M}_{K_{\mathrm{S}}}$ estimated by the KPCA method using the LAMOSTHipparcos training set, parameter errors induced by spectral noises and those by the method are estimated separately. Errors induced by spectral noises, as in the case of LSS-GAC DR1, are estimated by comparing results derived from duplicate observations made in different nights, and are estimated separately for giants $(\log g<3.5$ dex $)$ and dwarfs $(\log g>3.5$ dex $)$. The results are fitted with a second-order polynomial function of SNR, $T_{\text {eff }}$ and $[\mathrm{Fe} / \mathrm{H}]$,

$$
\begin{aligned}
\sigma= & c_{0}+c_{1} \times \mathrm{SNR}+c_{2} \times T_{\mathrm{eff}}+c_{3} \times[\mathrm{Fe} / \mathrm{H}]+c_{4} \times \mathrm{SNR}^{2} \\
& +c_{5} \times T_{\mathrm{eff}}^{2}+c_{6} \times[\mathrm{Fe} / \mathrm{H}]^{2}+c_{7} \times \mathrm{SNR} \times T_{\mathrm{eff}} \\
& +c_{8} \times \mathrm{SNR} \times[\mathrm{Fe} / \mathrm{H}]+c_{9} \times T_{\mathrm{eff}} \times[\mathrm{Fe} / \mathrm{H}]
\end{aligned}
$$

Here $\log g$ estimated with the weighted-mean algorithm is used to group stars into giants and dwarfs. Generally, the errors decrease significantly with increasing SNR, and hot or metal-poor stars have larger errors than cool or metal-rich ones.

The method errors are deduced from the residuals obtained by 
applying the method to spectra of the template library or the training sets themselves. Both the mean and dispersion of the residuals are calculated and fitted as functions of $T_{\text {eff }}$ and $[\mathrm{Fe} / \mathrm{H}]$, for dwarfs and giants separately. The mean reflects the bias induced by the method, and is thus corrected for for all target stars. While the dispersion is combined with the error induced by the spectral noises to yield the final value of error of the estimated parameter of concern. In doing so, a grid of method errors (mean and dispersion) is first created in the $T_{\text {eff }}-[\mathrm{Fe} / \mathrm{H}]$ plane, and for a given set of atmospheric parameters of a target star, the corresponding value of method error is interpolated from the grid.

For $\log g$ estimated with the KPCA method using the LAMOST-Kepler training set, as well as metal/elemental abundances $([\mathrm{M} / \mathrm{H}],[\mathrm{Fe} / \mathrm{H}],[\mathrm{C} / \mathrm{H}],[\mathrm{N} / \mathrm{H}],[\alpha / \mathrm{M}],[\alpha / \mathrm{Fe}])$ estimated using the LAMOST-APOGEE training set, since there are sufficiently large number of stars in common with the Kepler and APOGEE surveys that have not been included in the training samples, they can be used as the test samples (Xiang et al. 2017) to directly estimate the parameter errors. Given that no obvious trends with $T_{\text {eff }}$, $\log g$ and $[\mathrm{Fe} / \mathrm{H}]$ are seen, the errors are estimated as a function of spectral SNR only.

\subsection{Specific flags}

Specific flags are assigned to each star to better describe the quality of estimated parameters. A flag is assigned to describe the type of the best-matching template star. Based on the SIMBAD database (Wenger et al.2000), LSP3 template stars are divided into 38 groups as listed in Table 3 . While the majority template stars are normal (single) stars of $\mathrm{A} / \mathrm{F} / \mathrm{G} / \mathrm{K} / \mathrm{M}$ spectral types, there are also considerable numbers of spectroscopic binaries, double or multiple stars, variable stars, as well as of other rare types. The flag is set to help identify stars of specific type, although a careful analysis is essential to validate the results. The flag is an integer of value from 1 to 38, and is labeled respectively by 'TYPEFLAG_CHI2' and 'TYPEFLAG_CORR' for results based on the minimum $\chi^{2}$ and correlation matching algorithms (Xiang et al. 2015c).

The second flag describes the correlation coefficient for radial velocity estimation. Although LSS-GAC intends to target stars that are identified as point sources in the photometry catalogues, there are still some contaminations from extragalactic sources (e.g. galaxies, QSOs), for which LSP3 gives problematic radial velocities because the pipeline treats all input spectra as stellar. In addition, radial velocity estimates can be problematic for stars of unusual spectra (e.g. of emission line stars) or defective spectra (e.g. those seriously affected by cosmic rays and/or scatter light). In such cases, it is found that the peak correlation coefficient for radial velocity estimation is small compared to those of bulk stars. To mark those objects, a flag is assigned to each star in a way similar to the third flag in Xiang et al. (2015c), with the only difference that the current flag is set to be a positive float number, with negative ones replaced by 0.0. Experience suggests that one should treat the radial velocity cautiously if the flag has a value larger than 6.0. The flag is denoted by 'VR_FLAG' in the value-added catalogues. The peak correlation coefficient is also given by flag 'PEAK_CORR_COEFF'.

Similar to the second flag of Xiang et al. (2015c), a flag is used to describe anomalies in the minimum $\chi^{2}$ of the best-matching spectral template. This flag is designed for parameters estimated with the weighted-mean algorithm. Again, the current flag is set to be a positive float, with negative values replaced by 0.0 . The flag is
Table 3. Types of template stars.

\begin{tabular}{|c|c|c|}
\hline Flag & Type & $\mathrm{N}$ \\
\hline 1 & Normal star (single; AFGKM) & 757 \\
\hline 2 & Pre-main sequence star & 1 \\
\hline 3 & L/T-type star & 9 \\
\hline 4 & O/B-type star & 19 \\
\hline 5 & S star & 6 \\
\hline 6 & Carbon star & 5 \\
\hline 7 & Blue supergiant star & 6 \\
\hline 8 & Red super giant star & 3 \\
\hline 9 & Evolved supergiant star & 1 \\
\hline 10 & Horizontal branch (HB) Star (not include RCs) & 27 \\
\hline 11 & Asymptotic giant branch (AGB) Star & 6 \\
\hline 12 & Post-AGB star (proto-PN) & 4 \\
\hline 13 & Planetary nebula (PN) & 1 \\
\hline 14 & Star in nebula & 3 \\
\hline 15 & Emission line star & 2 \\
\hline 16 & Pulsating variable star & 5 \\
\hline 17 & Semi-regular pulsating star & 22 \\
\hline 18 & Classical Cepheid (delta Cep type) & 10 \\
\hline 19 & Variable star of beta Cep type & 4 \\
\hline 20 & Variable star of RR Lyr type & 5 \\
\hline 21 & Variable star of BY Dra type & 28 \\
\hline 22 & Variable star of RS CVn type & 12 \\
\hline 23 & Variable star of alpha2 CVn type & 23 \\
\hline 24 & Variable star of delta Sct type & 21 \\
\hline 25 & Variable star of RV Tau type & 1 \\
\hline 26 & Rotationally variable star & 4 \\
\hline 27 & T Tau-type star & 1 \\
\hline 28 & Flare star & 10 \\
\hline 29 & Peculiar star & 4 \\
\hline 30 & Long-period variable star & 13 \\
\hline 31 & Spectroscopic binary & 80 \\
\hline 32 & Eclipsing binary of Algol type (detached) & 7 \\
\hline 33 & Eclipsing binary of beta Lyr type (semi-detached) & 2 \\
\hline 34 & Symbiotic star & 2 \\
\hline 35 & Star with envelope of $\mathrm{CH}$ type & 7 \\
\hline 36 & Variable star (unclassfied) & 78 \\
\hline 37 & Double or multiple star & 79 \\
\hline 38 & WD (DA) & 3 \\
\hline
\end{tabular}

denoted by 'CHI2_FLAG'. The minimum $\chi^{2}$ of the best-matching template is presented as 'MIN_CHI2'.

Another flag is assigned to indicate the quality of parameters estimated with the KPCA method. It is defined as the maximum value of the kernel function, $d_{\mathrm{g}}$, which reflects the minimum distance between the target and training spectra (Xiang et al. 2017). The flag is a float of value between 0.0 and 1.0, with larger values indicating better parameter estimates. All KPCA estimates that have a $d_{\mathrm{g}}$ value smaller than 0.2 are not provided in the valueadded catalogues due to their low reliability. The flag is labeled respectively by 'DG_MILES_TM', 'DG_MILES_G', 'DG_HIP', 'DG_KEP', 'DG_APO', for parameters estimated with the aforementioned different training sets, (cf. Table 5).

\subsection{Recommended parameter values}

Since different methods have been employed to deduce parameters $T_{\text {eff }}, \log g,[\mathrm{Fe} / \mathrm{H}]$ and $[\alpha / \mathrm{Fe}]$, one needs to choose between parameter values yielded by different methods for the specific problems that she/he may want to address. For this purpose, it is necessary to 

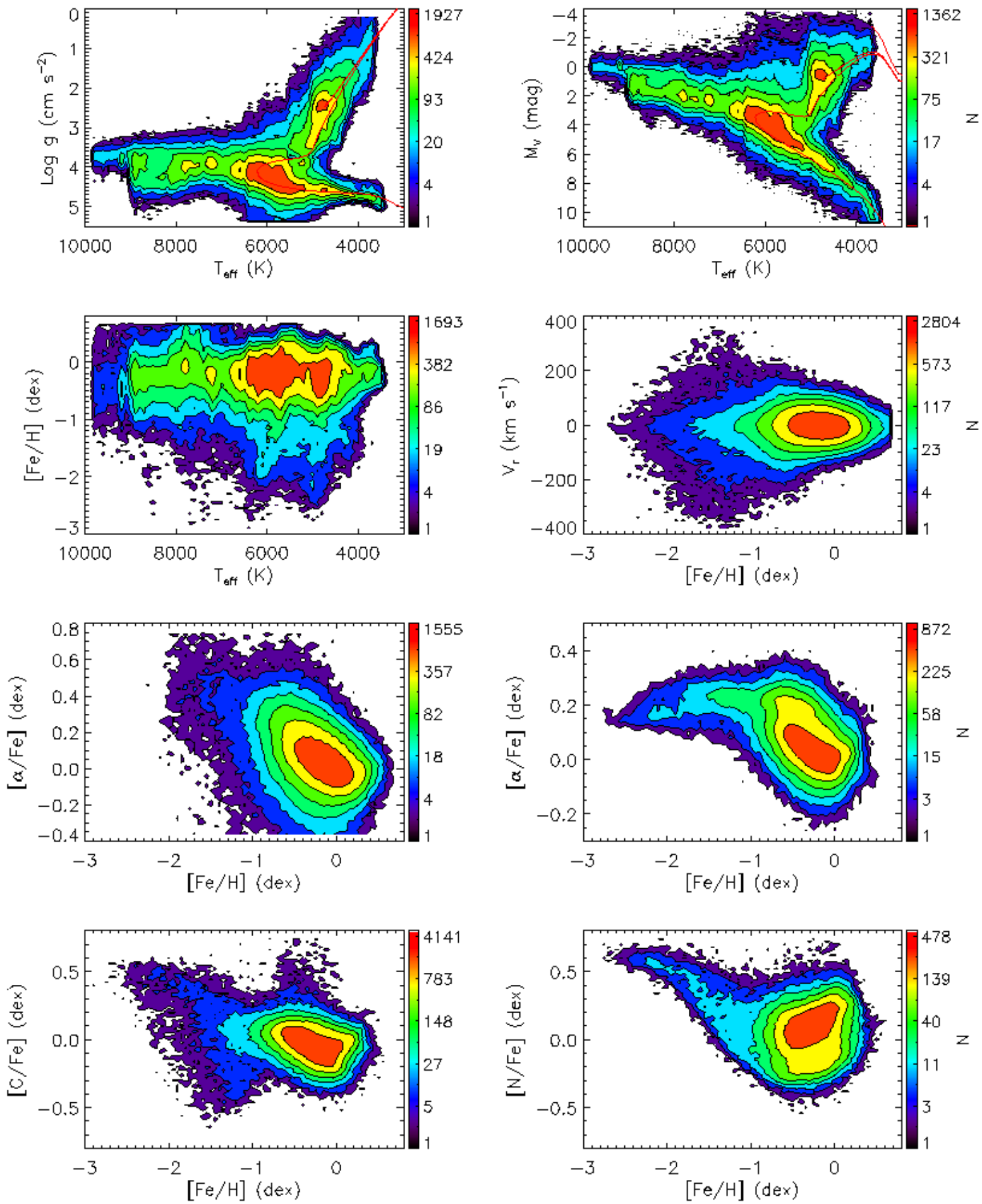

Figure 9. Contours of colour-coded stellar number density distributions in various planes of recommended parameters for the whole sample of stars in the value-added catalogues. Stellar isochrones in the $T_{\text {eff }}-\log g$ and $T_{\text {eff }}-\mathrm{M}_{V}$ plots are from Rosenfield et al. (2016) and have a solar metallicity and an age of 4.5 Gyr. The left and right panels of third row are $[\mathrm{Fe} / \mathrm{H}]-[\alpha / \mathrm{Fe}]$ plots showing results for dwarfs and giants, respectively. 

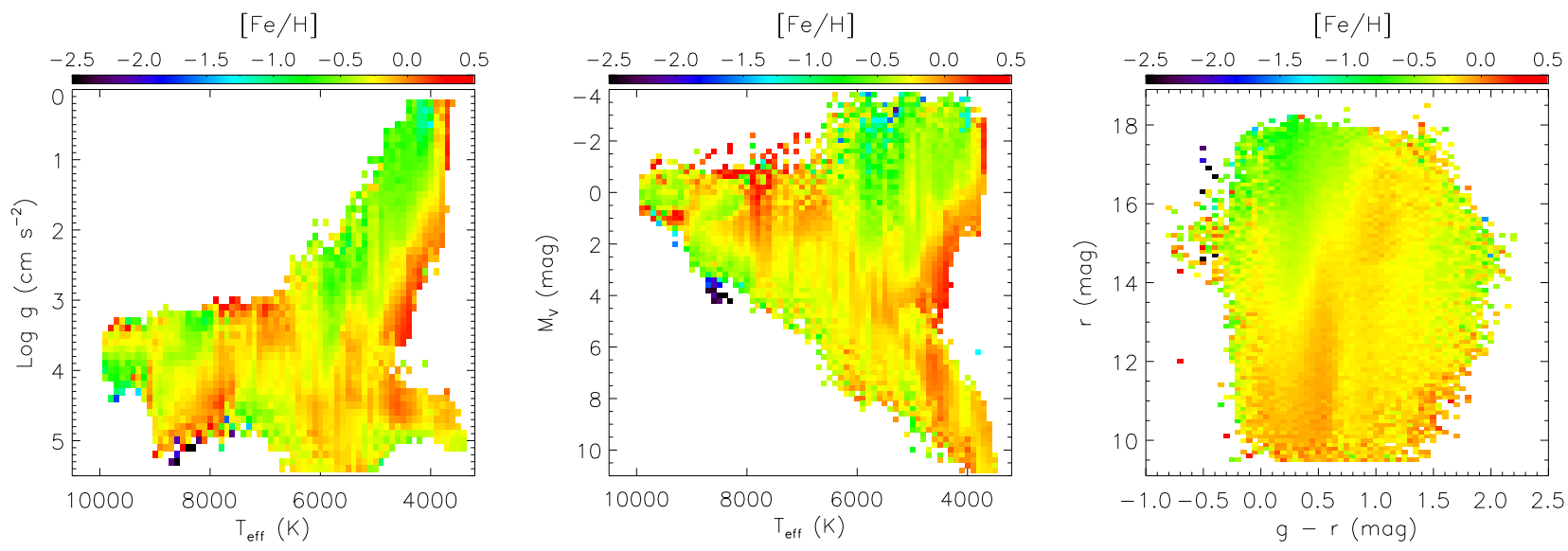

Figure 10. Distributions of sample stars in $T_{\text {eff }}-\log g, T_{\text {eff }}-\mathrm{M}_{V}$ and $(g-r, r)$ diagrams colour-coded by median metallicity, defined as the median value of stars in binsof $100 \mathrm{~K} \times 0.1 \mathrm{dex}$ in $T_{\text {eff }}-\log g, 100 \mathrm{~K} \times 0.2 \mathrm{mag}$ in $T_{\text {eff }}-\mathrm{M}_{V}$, and $0.05 \times 0.1 \mathrm{mag}$ in $(g-r, r)$ plane, respectively, for the three panels from left to right.

get to know both the advantages and limits of the individual methods employed.

For atmospheric parameters estimated with the weightedmean algorithm, the advantage is that the results are found to be relatively insensitive to spectral SNR compared to results yielded by other methods. This implies that for low SNRs (e.g. $<20$ ), results from the weighted-mean method are more robust and should be preferred. A disadvantage of the method is that the resultant parameters suffer from some systematics, as described above. The advantage of parameters estimated with the KPCA method is that they are much less affected by systematics, and thus more accurate than the weighted-mean values given sufficiently high spectral SNRs. A limitation is that they are available for limited ranges of parameters only, and, in addition, suffer from relatively large random errors at low SNRs.

We provide a recommended set of parameters $\left\{T_{\mathrm{eff}}, \log g\right.$, $\left.\mathrm{M}_{V}, \mathrm{M}_{K_{\mathrm{s}}},[\mathrm{Fe} / \mathrm{H}],[\alpha / \mathrm{Fe}]\right\}$ based on the above considerations. Table 4 lists the adopted methods for the recommended parameters and their effective parameter ranges. We emphasize that the recommended parameters are not necessarily the most accurate/precise ones. For example, although $[\mathrm{Fe} / \mathrm{H}]$ values estimated with the KPCA method using the MILES library are adopted as the recommended values, those estimated using the LAMOST-APOGEE training set are in fact more precise. Values of $T_{\text {eff }}$ derived with the weighted-mean method are robust, but suffer from clustering effect, and are thus weakly clumped in the parameter space on the scale of a few tens Kelvin. For $[\alpha / \mathrm{Fe}]$, considering that there are systematic differences between the template matching and KPCA results (Xiang et al. 2017), our recommendation will lead to some level of inconsistency between the results for giants and dwarfs. For $\mathrm{M}_{V}$ and $\mathrm{M}_{K_{\mathrm{s}}}$, absolute magnitudes yielded using $300 \mathrm{PCs}$ are adopted as the recommend values, but if the targets of interest are mainly composed of stars with low spectral SNRs $(<30)$, one would better use results from 100 PCs. In addition, because a cut of 0.2 is set for the maximum value of the kernel function, $d_{g}$, to safeguard robust parameter estimates with the KPCA method Xiang et al. 2017), about 18 per cent of the sample stars (mostly with very low SNRs) have no parameter estimates with the KPCA method. For those stars, the recommended parameters are not available. Rather than adopting the recommended values, we encourage users to make their own choice based on their specific problems under consideration.

For $[\mathrm{M} / \mathrm{H}],[\alpha / \mathrm{M}],[\mathrm{C} / \mathrm{H}]$ and $[\mathrm{N} / \mathrm{H}]$, since at the moment there are only one set of measurements, i.e. those with the KPCA method, they are simply adopted as the recommended values. Note that $[\mathrm{M} / \mathrm{H}],[\alpha / \mathrm{M}],[\mathrm{C} / \mathrm{H}]$ and $[\mathrm{N} / \mathrm{H}]$ are available only for stars of $T_{\text {eff }}<5600 \mathrm{~K}$ and $\log g<3.8$ dex. Here $T_{\text {eff }}$ refers to that estimated with the weighted-mean method, while $\log g$ is either estimated with the weighted-mean method or by the KPCA method using the MILES stars as the training set.

Fig. 9 plots the stellar number density distributions in various planes of recommended parameters. In the $T_{\text {eff }}-\log g$ and $T_{\text {eff }}-\mathrm{M}_{V}$ planes, the stars show two prominent clumps, one composed of FGK dwarfs and another of red clump stars. The compact contours of red clump stars indicate a high precision of the estimated parameters. Trajectories of stars in those planes are well consistent with the theoretical isochrones. Stars of $[\mathrm{Fe} / \mathrm{H}]>-1.0$ dex dominate the sample, indicating that most of the stars belong to the Galactic disk. The fraction of stars with $[\mathrm{Fe} / \mathrm{H}]<-1.0$ dex is only 1.8 per cent. Given the large difference between the numbers of metal-rich and metal-poor stars, even a small fraction of those metal-rich stars whose $[\mathrm{Fe} / \mathrm{H}]$ estimates may have suffered large uncertainties could seriously contaminate the sample of metal-poor stars. This seems to have led to the appearance of a kinematically cold but metal-poor population in the $[\mathrm{Fe} / \mathrm{H}]-V_{\mathrm{r}}$ plane. One thus needs to be very careful when studying the metal-poor populations using this diskstar dominant sample. Comparison of $[\mathrm{Fe} / \mathrm{H}]$ values derived with the weighted-mean and KPCA methods may help identify potential contaminators. Exercise shows that one excludes stars whose $\mathrm{KPCA}[\mathrm{Fe} / \mathrm{H}]$ estimates are smaller than the weighted-mean values by 0.3 dex or replace the estimates by the latter values, the artificial populations of cold, metal-poor stars in the $[\mathrm{Fe} / \mathrm{H}]-V_{\mathrm{r}}$ plane is largely disappears.

Fig. 9 also shows that more metal-poor stars generally have higher $[\alpha / \mathrm{Fe}]$ values. This is true for both dwarfs and giants, a natural consequence of the Galactic chemical evolution. For both dwarfs and giants, $[\alpha / \mathrm{Fe}]$ values for stars of solar metallicity are close to zero. Nevertheless, $[\alpha / \mathrm{Fe}]$ values of dwarfs and giants show different patterns. Dwarfs cover a wider range of $[\alpha / \mathrm{Fe}]$ values than giants. There are very few giant stars of $[\alpha / \mathrm{Fe}]<$ 
-0.2 dex or $[\alpha / \mathrm{Fe}]>0.3$ dex. In contrast, there are quite a number of dwarfs of $[\alpha / \mathrm{Fe}]<-0.3$ dex or $[\alpha / \mathrm{Fe}]>0.4$ dex. Moreover, besides the dominant thin disk sequence in the $[\alpha / \mathrm{Fe}]-[\mathrm{Fe} / \mathrm{H}]$ plane, giants exhibit also an extra, weaker sequence of thick disk stars of a $[\alpha / \mathrm{Fe}]$ value of $\sim 0.2$ dex. This thick disk sequence is not seen in dwarfs. The larger random errors (Fig. 13) of $[\alpha / \mathrm{Fe}]$ estimates for dwarfs are definitively an important reason for the absence of this sequence. Also, giants probe a larger volume than dwarfs as they are brighter. Thus the giant sample contains more thick disk stars, whereas the dwarf sample is dominated by the local thin disk stars and young, hotter stars in the outer thin disk. In addition, and maybe more importantly, since different algorithms, spectral templates and wavelength ranges are used for $[\alpha / \mathrm{Fe}]$ estimation for dwarfs and giants, and the results have not been calibrated externally using data for example from high resolution spectroscopy, systematic differences and errors may well be hided in the $[\alpha / \mathrm{Fe}]$ estimates of dwarfs and/or giants. A calibration of $[\alpha / \mathrm{Fe}]$ estimates is an essential and urgent task for the future.

Fig. 10 plots distributions of stars in the $T_{\text {eff }}-\log g, T_{\text {eff }}-$ $\mathrm{M}_{V}$ and $(g-r, r)$ planes colour-coded by $[\mathrm{Fe} / \mathrm{H}]$. The figure shows that amongst the cool stars, as expected, metal-rich ones generally have lower temperatures than those metal-poor ones. However, for hot stars, the derived metallicities show some strange patterns in the $T_{\text {eff }}-\log g$ plane that are not fully understood yet. A possible cause could be the inadequacy of parameter coverage of the MILES spectral library at higher temperatures. A further, detailed examination of $\log g$ estimates utilizing LAMOST-TGAS common stars, in particular for hot stars, is underway. On the whole, the trends of $[\mathrm{Fe} / \mathrm{H}]$ seen in the $T_{\text {eff }}-\mathrm{M}_{V}$ and the $(g-r, r)$ planes seem to be reasonable. The brighter side of the $(g-r, r)$ diagram is dominated by metal-rich stars as they represent a local, thin disk sample. On the other hand, at the fainter side of the diagram, one sees metalrich stars are in general redder while the metal-poor ones are bluer. Again, this is what one would expect.

Fig. 11 plots error distributions of recommended parameter estimates. Spectral SNR is the main factor that determines the amount of parameter error, and stars of different spectral types have also different parameter errors. A detailed analysis of how the parameter errors depends on spectral SNR and stellar parameters can be found in Xiang et al. (2015a, 2017). Most of the stars have a radial velocity error of a few $\mathrm{km} \mathrm{s}^{-1}$. However, some of them, mostly hot stars of low SNRs, have errors reaching $20 \mathrm{~km} \mathrm{~s}^{-1}$. Temperature errors are around $100 \mathrm{~K}$ for most stars, but those of hot ones reach $400-600 \mathrm{~K}$. The large temperature errors for hot stars are mainly caused by the small number and large parameter uncertainties of hot stars in the MILES library. Similar trend is seen in $[\mathrm{Fe} / \mathrm{H}] \mathrm{er}-$ rors, where most stars have an error between 0.1 and 0.2 dex. However, the error distribution exhibits a second peak at 0.5-0.6 dex, mainly contributed by hot stars. Errors of $\log g$ typically have values of 0.1-0.2 dex, with a small fraction reaching 0.3-0.4 dex. Errors of $\mathrm{M}_{V}$ can be as small as 0.2 mag at high spectral SNRs, but reach $0.8-1.0 \mathrm{mag}$ at low SNRs $(\sim 10)$. $\mathrm{M}_{V}$ values estimated using $100 \mathrm{PCs}$ have larger errors compared to those deduced using $300 \mathrm{PCs}$, but they are less sensitive to SNR, and have errors of 0.6 -0.8 mag at low SNRs $(\sim 10)$. Errors of $\mathrm{M}_{K_{\mathrm{S}}}$ have similar trends. Errors in $[\alpha / \mathrm{Fe}]$ show two populations. The lower one is of giants, which has values of 0.02-0.09 dex. The higher one is of dwarfs, and has values of $0.09-0.25$ dex, peaking around 0.1 dex. Note that as discussed above, since the results are not calibrated by independent, external datasets, there could be systematic uncertainties in $[\alpha / \mathrm{Fe}]$ estimates that have not been included in the current error estimates. This is particularly true for giant stars, for which the quoted errors
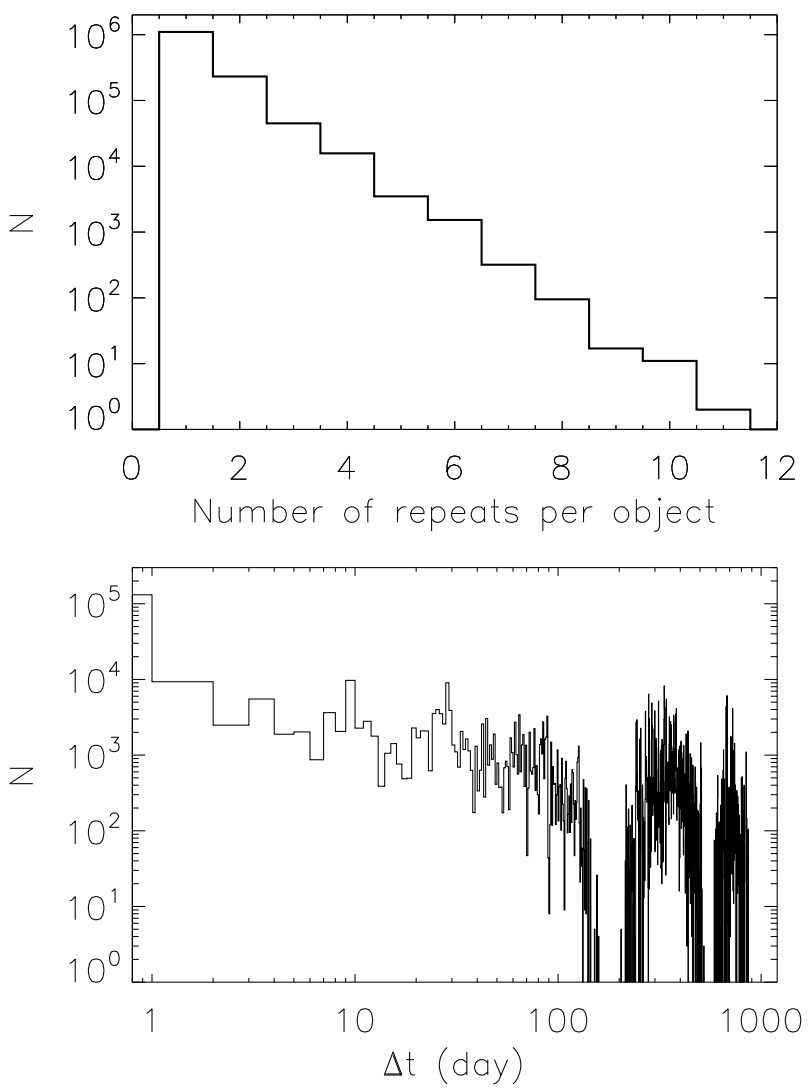

Figure 13. Upper: Stellar numbers as a function of number of repeat observations per object. Lower: Distribution of time separation for repeat observations.

are very small. Errors of $[\mathrm{C} / \mathrm{H}]$ and $[\mathrm{N} / \mathrm{H}]$ estimates range from 0.05 to 0.20 dex, with most of them smaller than 0.15 dex.

A comparison of recommended stellar atmospheric parameters with LSS-GAC DR1 for the whole LSS-GAC DR1 sample is shown in Fig. 12. The figure shows that $T_{\text {eff }}$ estimates from the two data releases are quite consistent. For $\log g$, the plot shows significant patterns. Similar patterns are seen by comparing LSS-GAC DR1 $\log g$ estimates with asteroseismic measurements (Ren et al. 2016). The causes of such patterns are discussed in Xiang et al. (2017), and are essentially due to the inadequacies of $\log g$ estimates in LSS-GAC DR1 ones. On the whole, new [Fe/H] estimates show good correlation with those of LSS-GAC DR1. The standard deviation of the two sets of $[\mathrm{Fe} / \mathrm{H}]$ estimates of the whole sample is 0.15 dex. However, the current estimates are 0.05 dex systematically lower than LSS-GAC DR1. There is also a group of stars whose new $[\mathrm{Fe} / \mathrm{H}]$ values are significantly higher than those of LSS-GAC DR1. In addition, the sharp boundary seen in the LSSGAC DR1 results now disappears. Again, the causes of these differences are discussed in Xiang et al. (2017), and are mainly due to the inadequacies of $[\mathrm{Fe} / \mathrm{H}]$ estimates in LSS-GAC DR1.

\section{REPEAT OBSERVATIONS}

About 28 per cent of stars in the value-added catalogues have repeat observations. The repeat observations are valuable for various purposes, including (1) time-domain spectroscopy; (2) robustness 
Table 4. Adopted methods and their effective parameter ranges for stellar parameter determinations.

\begin{tabular}{|c|c|c|}
\hline Parameter & Method & Effective range \\
\hline \multicolumn{3}{|l|}{ Effective temperature } \\
\hline$T_{\text {eff_1 }}$ & Weighted-mean template matching & All \\
\hline$T_{\text {eff_2 }}$ & KPCA with the MILES training set & $T_{\text {eff__}} 1<10000 \mathrm{~K}$ \\
\hline$T_{\text {eff }}($ recommended $)$ & $T_{\text {eff__}} 1$ & All \\
\hline \multicolumn{3}{|l|}{ Surface gravity } \\
\hline Logg_1 & Weighted-mean template matching & All \\
\hline Logg_2 & KPCA with the MILES training set & $T_{\text {eff__ }} 1<10000 \mathrm{~K}, \operatorname{Logg} \_1>3.0 \mathrm{dex}$ \\
\hline Logg_3 & KPCA with the LAMOST-Kepler training set & $T_{\text {eff_}} 1<5600 \mathrm{~K}$, Logg_1 $1<3.8 \mathrm{dex}$ or Logg_2 $<3.8 \mathrm{dex}$ \\
\hline \multirow[t]{3}{*}{ Logg (recommended) } & Logg_1 & $T_{\text {eff_} \_1}>10000 \mathrm{~K}$ \\
\hline & Logg_3 & $T_{\text {eff_}} 1<5600 \mathrm{~K}$, Logg_1 $<3.8$ dex or Logg_2 $<3.8 \mathrm{dex}$ \\
\hline & Logg_2 & Otherwise \\
\hline \multicolumn{3}{|l|}{ Metallicity } \\
\hline$[\mathrm{Fe} / \mathrm{H}]_{-} 1$ & Weighted-mean template matching & All \\
\hline$[\mathrm{Fe} / \mathrm{H}] \_2$ & KPCA with the MILES training set & $T_{\text {eff_1 }}<10000 \mathrm{~K}$ \\
\hline$[\mathrm{Fe} / \mathrm{H}] \_3$ & KPCA with the LAMOST-APOGEE training set & $T_{\text {eff_}} 1<5600 \mathrm{~K}$, Logg_ $1<3.8$ dex or Logg_2 $<3.8 \mathrm{dex}$ \\
\hline \multirow[t]{2}{*}[\mathrm{Fe}/\mathrm{H}]{$($ recommended $)$} & {$[\mathrm{Fe} / \mathrm{H}] \_1$} & $T_{\text {eff_ } \_1}>10000 \mathrm{~K}$ \\
\hline & {$[\mathrm{Fe} / \mathrm{H}] \_2$} & Otherwise \\
\hline \multicolumn{3}{|c|}{$\alpha$-element to iron abundance ratio } \\
\hline$[\alpha / \mathrm{Fe}] \_1$ & $\begin{array}{l}\text { Template matching using spectra of } \\
3900-3980,4400-4600 \text { and } 5000-5300 \AA\end{array}$ & $4000<T_{\text {eff__ }} 1<8000 \mathrm{~K}$ \\
\hline$[\alpha / \mathrm{Fe}] \_2$ & $\begin{array}{l}\text { Template matching using spectra of } \\
4400-4600 \text { and } 5000-5300 \AA\end{array}$ & $4000<T_{\text {eff__ }} 1<8000 \mathrm{~K}$ \\
\hline \multirow{3}{*}{$\begin{array}{l}{[\alpha / \mathrm{Fe}] \_3} \\
{[\alpha / \mathrm{Fe}](\text { recommended })}\end{array}$} & KPCA with the LAMOST-APOGEE training set & $T_{\text {eff_} \_} 1<5600 \mathrm{~K}$, Logg_ $1<3.8$ dex or Logg_2 $<3.8$ dex \\
\hline & {$[\alpha / \mathrm{Fe}] \_3$} & $T_{\text {eff_}} 1<5600 \mathrm{~K}$, Logg_ $1<3.8$ dex or Logg_2 $2<3.8 \mathrm{dex}$ \\
\hline & {$[\alpha / \mathrm{Fe}] \_1$} & Otherwise \\
\hline \multicolumn{3}{|c|}{$\begin{array}{l}\text { Other elemental abundances } \\
{[\mathrm{M} / \mathrm{H}]}\end{array}$} \\
\hline$[\alpha / \mathrm{M}]$ & KPCA with the LAMOST-APOGEE training set & $T_{\text {eff_}} 1<5600 \mathrm{~K}$, Logg_ $1<3.8$ dex or Logg_2 $<3.8 \mathrm{dex}$ \\
\hline \multicolumn{3}{|r|}{ 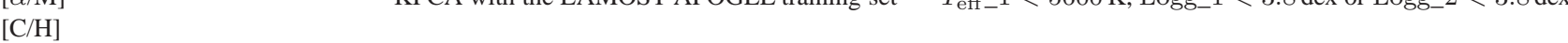 } \\
\hline \multicolumn{3}{|l|}{$[\mathrm{N} / \mathrm{H}]$} \\
\hline \multicolumn{3}{|l|}{ Absolute magnitudes } \\
\hline $\mathrm{M}_{V \_} 1$ & KPCA with the LAMOST-Hipparcos training set & \\
\hline $\mathrm{M}_{K_{\mathrm{s}-}-1}$ & using $300 \mathrm{PCs}$ & 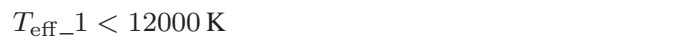 \\
\hline $\mathrm{M}_{V \_}$ & KPCA with the LAMOST-Hipparcos training set & \\
\hline $\mathrm{M}_{K_{\mathrm{s}}-2}$ & using $100 \mathrm{PCs}$ & $T_{\text {eff_1 }}<12000 \mathrm{~K}$ \\
\hline $\mathrm{M}_{V}$ (recommended) & $\mathrm{M}_{V \_} 1$ & \\
\hline $\mathrm{M}_{K_{\mathrm{S}}}($ recommended $)$ & $\mathrm{M}_{K_{\mathrm{s}-}-1}$ & 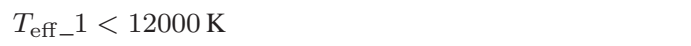 \\
\hline
\end{tabular}

check of parameter estimates; (3) improvement of stellar parameter estimates by averaging repeat measurements, etc. Fig. 13 plots the distribution of number of stars as a function of number of repeat observations per object, as well as the distribution of time separations of repeat observations. Stellar number decreases approximately linearly with increasing number of repeat observations. There are 95350 unique stars that are observed 3 times or more, and 5460 unique stars observed 5 times or more. Time separation covers a range from a few hours to nearly 1000 days. Most repeat observations occurred in the same night, when different plates that shared the same central bright stars or adjacent plates that had overlapping fields of views were observed. Note that to better present the data, the horizontal axis of the figure has been chosen to artificially start from 0.8 days, but the real time separation in the first bin was smaller than 4 hours due to hour angle limitations of LAMOST (Cui et al. 2012). Note also that here repeat observations refer to those taken with different plates. In fact, each plate observed usually has 2-3 exposures. The current analysis is based on co-added spectra of those adjacent individual exposures. Further, independent treatment of the individual exposures of each plate observed in the future will significantly increase the repeat observation rate in the sense that every star targeted has at least one repeat observation, separated by tens of minutes.

Some systematic studies utilizing repeat observations are underway. As an example, Fig. 14 shows the spectra in the 3900$4400 \AA$ wavelength range of two stars with repeat observations. The data clearly show shifts of spectral line positions, thus radial velocity variations. The first example has an amplitude of radial velocity variations larger than $180 \mathrm{~km} \mathrm{~s}^{-1}$. The offsets of repeat observations from the first one are respectively 3.99411, 4.02374, 4.04600, 72.85550 and 76.767793 days. Effective temperatures estimated from the six observations are respectively 4931, $5192,5150,5182,5507$ and $4953 \mathrm{~K}, \mathrm{M}_{V}$ absolute magnitudes $6.10,5.89,5.82,5.43,5.42$ and $6.56 \mathrm{mag}$, and $[\mathrm{Fe} / \mathrm{H}]$ metallicities $-0.22,-0.33,-0.24,-0.34,-0.15$ and -0.07 dex. The second example star has a radial velocity variation amplitude larger 

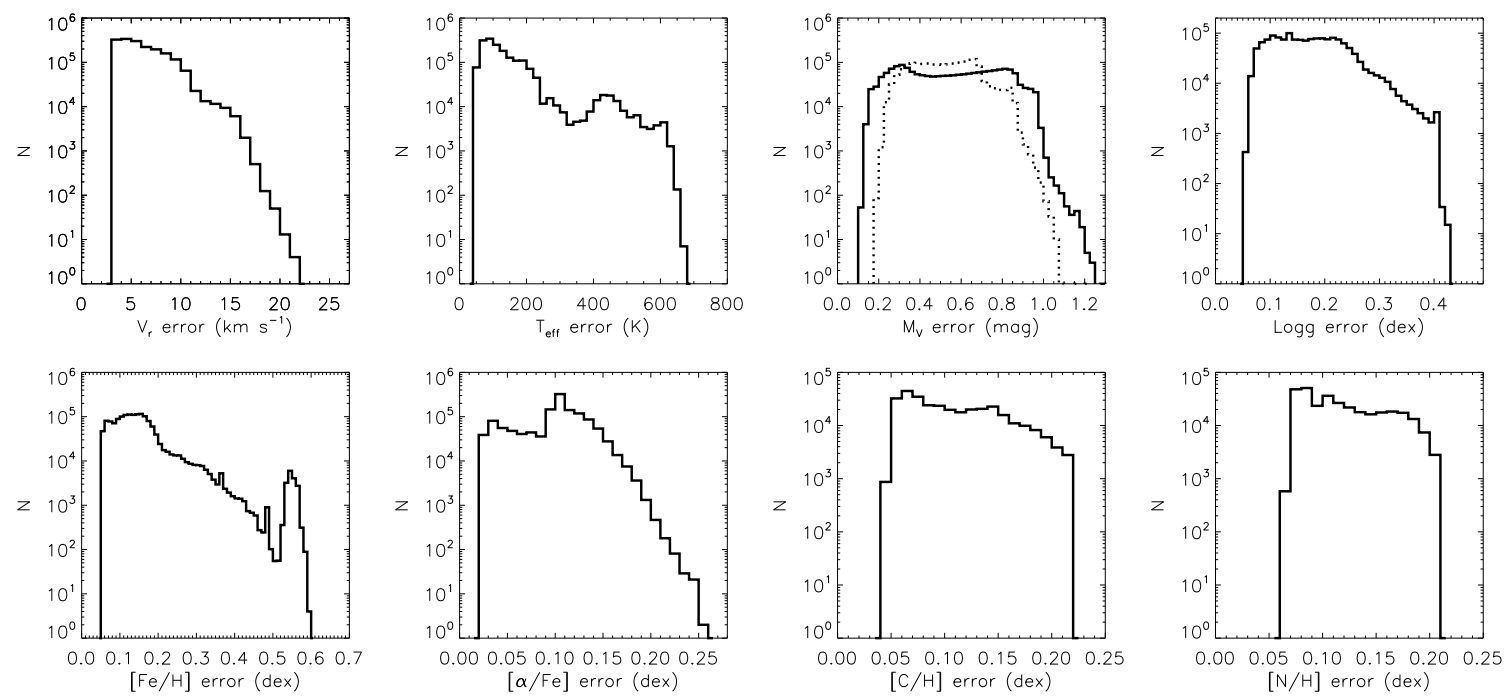

Figure 11. Error distributions of recommended parameter estimates. For $\mathrm{M}_{V}$, error distribution of the other set of $\mathrm{M}_{V}$ estimates deduced using 100 PCs is shown by dotted line.
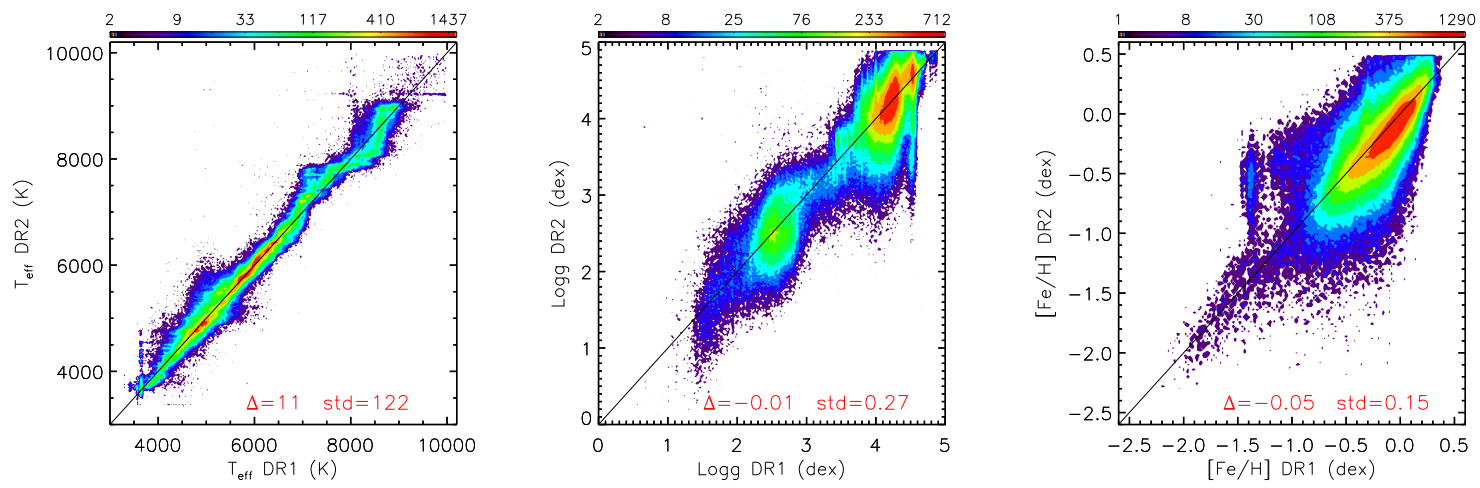

Figure 12. Comparison of the new recommended stellar atmospheric parameters with those of LSS-GAC DR1. Mean and standard deviations of the parameter differences between DR2 and DR1 are marked in the plot.

than $60 \mathrm{~km} \mathrm{~s}^{-1}$. The offsets of repeat observations from the first one are respectively $0.03447,346.02393,346.06302,374.90549$, 374.94406 and 374.98718 days. $T_{\text {eff }}$ estimates from the seven observations are respectively 5349, 5340, 5046, 5258, 5512, 5512 and $5526 \mathrm{~K}$, the $\mathrm{M}_{V}$ absolute magnitudes 4.47, 4.29, 4.62, 4.49, $4.74,4.79$ and $4.76 \mathrm{mag}$, and $[\mathrm{Fe} / \mathrm{H}]$ metallicities $-0.11,-0.05$, $0.03,0.00,-0.04,-0.03$ and -0.04 dex. It seems that in additions to radial velocity variations, the two stars also exhibit significant variations in effective temperature and absolute magnitude. Further analyses are however required to reveal the nature of the variations.

A detailed robustness examination on the LSP3 stellar parameters using repeat observations can be found in Xiang et al. 2015a) for radial velocity measurements and atmospheric parameter determinations with the weighted-mean method, and in Xiang et al. (2017) for atmospheric parameters estimated with the KPCA method. Fig. 15 plots the dispersions of differences of recommended parameters deduced from repeat observations that have comparable SNRs (20 per cent) as a function of SNR. The dispersions represent random errors of the parameters induced by spectral noises. For this purpose, values of dispersions shown in the figure have been divided by square root of 2 . Results for giants, hot $\left(T_{\text {eff }}>7000 \mathrm{~K}\right)$ and cool $\left(T_{\text {eff }}<7000 \mathrm{~K}\right)$ dwarfs are shown separately. Generally, hot dwarfs have larger random errors in $V_{\mathrm{r}}, T_{\mathrm{eff}}$, $[\mathrm{Fe} / \mathrm{H}]$ and $[\alpha / \mathrm{Fe}]$ estimates. For all parameters, the random errors are quite sensitive to SNR. Given a SNR higher than 100, random errors of radial velocities induced by spectral noises for cool stars can be as small as $1-2 \mathrm{~km} \mathrm{~s}^{-1}$, and the values are $3-4 \mathrm{~km} \mathrm{~s}^{-1}$ for hot stars. For a SNR of 10 , the errors increase to $5-10 \mathrm{~km} \mathrm{~s}^{-1}$ for cool stars and $20 \mathrm{~km} \mathrm{~s}^{-1}$ for hot stars. Note that the final error estimates of $V_{\mathrm{r}}$ have been set a minimum value of $3 \mathrm{~km} \mathrm{~s}^{-1}$ to account for the potential systematic uncertainties, which is $\sim 2 \mathrm{~km} \mathrm{~s}^{-1}$ as estimated via external examinations using the LAMOST-APOGEE common stars. As introduced in Section 4.4, for all stellar parameters, these random errors and their trends of variations with spectral $\mathrm{SNR}, T_{\text {eff }}$ and $[\mathrm{Fe} / \mathrm{H}]$ have been incorporated into the final error estimates, which include also uncertainties induced by the inadequacies of the parameter estimation methods for both giants and dwarfs. 

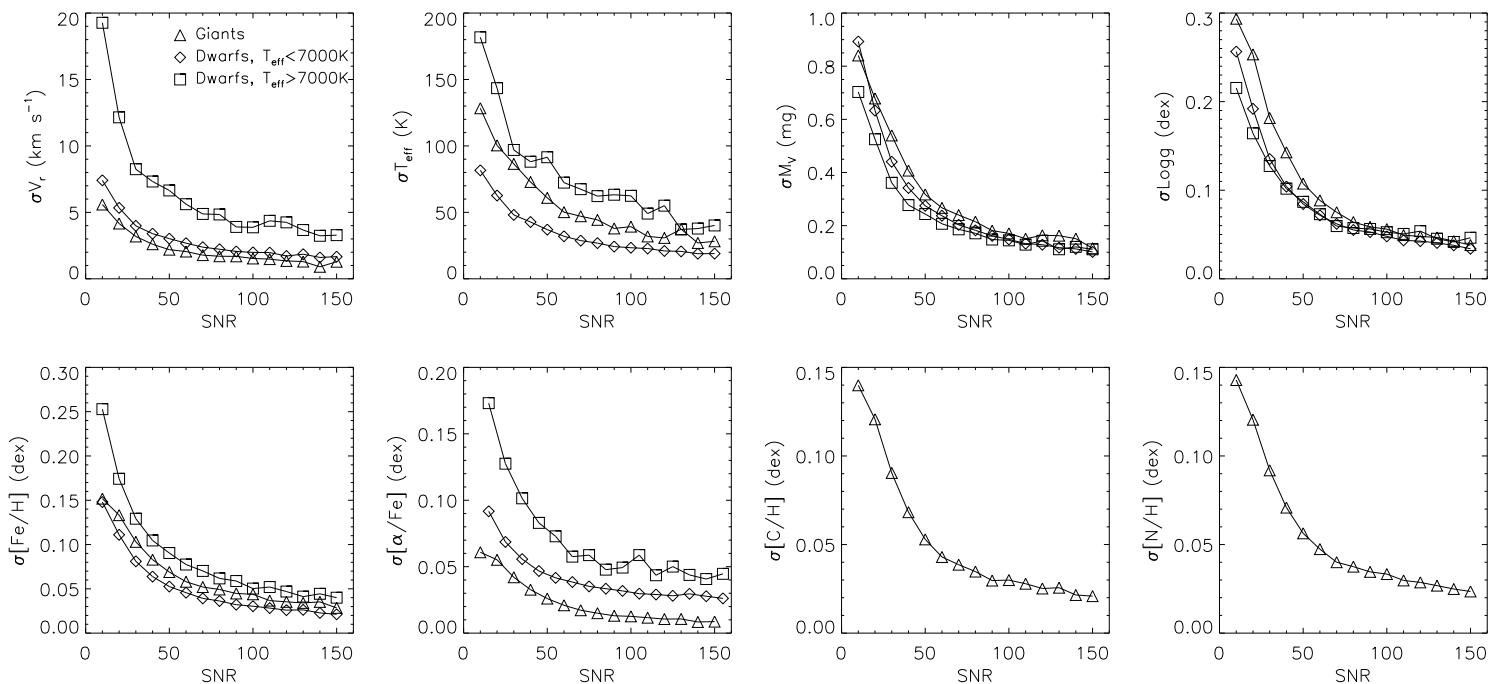

Figure 15. Random errors of recommend parameters as a function of spectral SNR as deduced by comparing results from repeat observations. Results for giants, hot and cool dwarfs are shown separately.

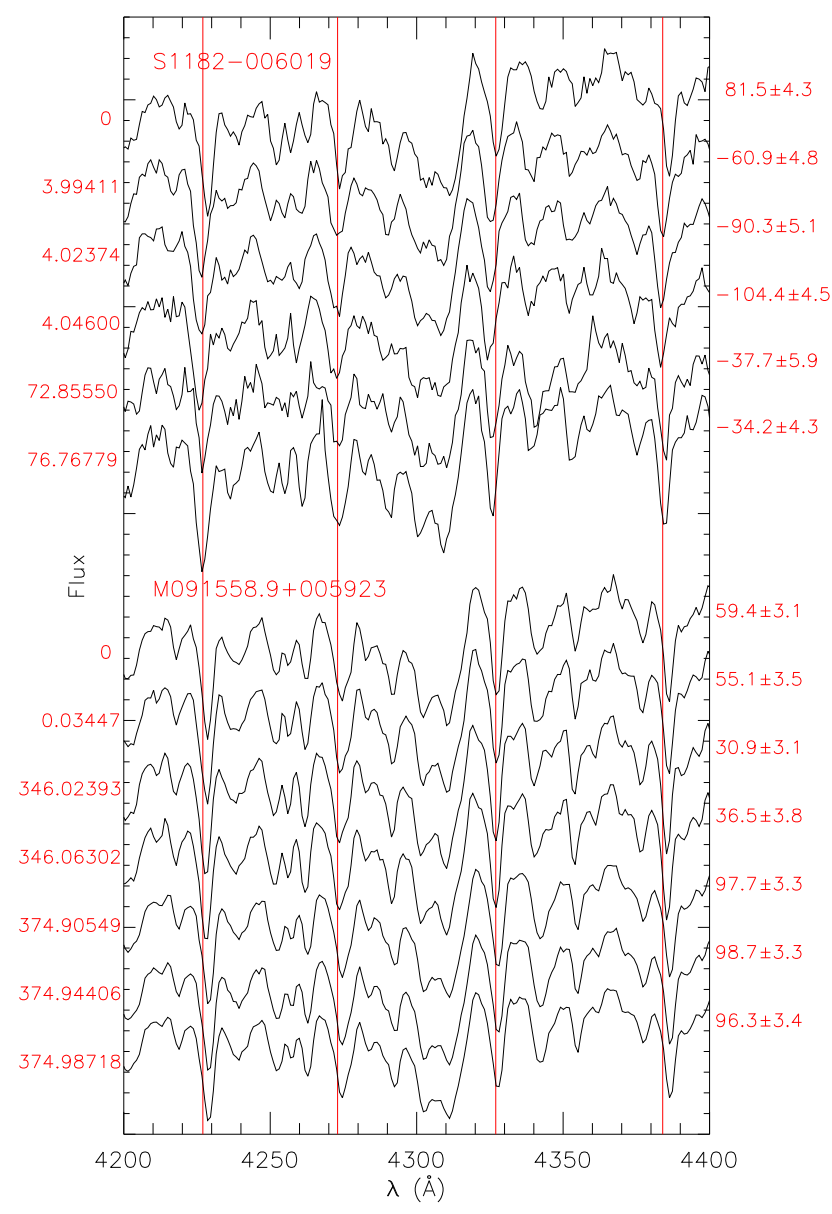

Figure 14. Examples of spectra of wavelength range 4200-4400尺 of two stars with repeat observations. Numbers on the left are JD offsets respect to the first observation, while numbers on the right are radial velocity measurements. Object ID from the input catalogues are marked on the plot.

\section{EXTINCTION \& DISTANCE}

\subsection{Extinction}

Similar to LSS-GAC DR1 (Yuan et al. 2015), various techniques are employed to estimate the interstellar reddening toward the individual stars. The methods include the star-pair technique, comparing observed colours with synthetic ones from stellar model atmospheres, as well as a method based on the stellar colour loci.

The star-pair method assumes that stars with identical stellar atmospheric parameters $\left(T_{\text {eff }}, \log g,[\mathrm{Fe} / \mathrm{H}]\right)$ have the same intrinsic colours. Thus for a reddened star, its intrinsic colours can be inferred from its pairs/counterparts with either nil or well known extinction (Yuan et al. 2015). The method is straightforward, and moreover, free from stellar model atmospheres. To derive extinction with this method, a control sample with nil or well known extinction is inherited from LSS-GAC DR1. The control sample contains low extinction stars whose $E(B-V)$ values can be well approximated by the reddening map of Schlegel, Finkbeiner \& Davis (1998, hereafter SFD98). For a given target star, the $E(B-V)$ is then derived by fitting $g-r, r-i, i-J, J-H$ and $H-K_{\mathrm{s}}$ optical to the infrared colours simultaneously by the corresponding values of the pair stars in the control sample, assuming the universal extinction coefficients of Yuan, Liu \& Xiang (2013). When available, $g, r, i$-band magnitudes from the XSTPS-GAC survey (Zhang et al. 2013, 2014; Liu et al. 2014) are used, otherwise they are taken from the APASS survey (Munari et al. 2014), or from the SDSS survey (DR8; Aihara et al. 2011) for a few B/M/F plates of high Galactic latitudes. $J, H, K_{\mathrm{s}}$ magnitudes are from the 2MASS survey (Skrutskie et al. 2006). There are a few ( $\sim 3-5$ per cent) stars for which different colours yield quite different reddenings. For those stars, either the photometry or the stellar atmospheric parameters, or both, are problematic. It is found that in such cases, $E(B-V)$ is often overestimated by a significant amount. To account for this, if $g-r$ and $r-i$ colours of a given star yield reddening of different signs and yield values differed by more than $0.2 \mathrm{mag}$, the resultant $E(B-V)$ estimate is discarded. The corresponding entry in the value-added catalogues are set to -9 . Comparison with the SFD98 reddening map for high Galactic latitude regions of 
low extinction illustrates a typical uncertainty of 0.036 mag for $E(B-V)$ thus estimated (Fig. 16), which is similar to that of LSS-GAC DR1. Note that we use the recommended atmospheric parameters for reddening estimation. Different results are expected if different sets of atmospheric parameters are adopted. Nevertheless, given the good precision of the current parameter estimates, the differences are small. For example, $E(B-V)$ values derived using atmospheric parameters estimated with the weighted-mean algorithm differ from the default results by a dispersion of only $\sim 0.01$ mag. $E(B-V)$ thus estimated is denoted 'EBV_SP' in the value-added catalogues.

$E(B-V)$ values have also been determined by comparing photometric colours with synthetic ones. In doing so, a grid of synthetic colours is first constructed by convolving the synthetic spectra of Castelli \& Kurucz (2004) with the transmission curves of filters for the photometric systems of SDSS 3 (Gunn et al. 1998; Doi et al. 2010) and 2MASS4 (Milligan, Cranton \& Skrutskie 1996). Synthetic colours for a star of given atmospheric parameters $T_{\text {eff }}, \log g$ and $[\mathrm{Fe} / \mathrm{H}]$ are then deduced by linearly interpolating the grid. $E(B-V)$ is then estimated as the weighted mean of values inferred from the individual colours, weighted by the reddening coefficients of Yuan, Liu \& Xiang (2013). As Fig. 16 shows, $E(B-V)$ values thus derived have a precision of about $0.03 \mathrm{mag}$, but are found to be systematically lower than the values given by either the star-pair method or by the SFD98 map, by $0.01-0.02$ mag. The underestimation is likely to be caused by the slight different temperature scales between the LSP3 and Kurucz atmospheric models. As pointed out above, LSP3 temperatures are calibrated to the metallicity-dependent colour-temperature relations of Huang et al. (2015a), constructed using a sample of stars with $T_{\text {eff }}$ estimated directly from angular diameters measured with interferometry and the trigonometric parallaxes from the Hipparcos satellite. It is found that the relations of Huang et al. (2015a) yield temperatures generally $\sim 100 \mathrm{~K}$ lower than earlier results in the literature. $E(B-V)$ estimated with this method is denoted 'EBV_MOD' in the catalogues.

Finally, extinction values deduced from multi-band photometry by fitting the stellar colour loci (Chen et al. 2014) are also included in the catalogues. Results from this method are currently only available for stars within the footprint and magnitude range of XSTPS-GAC, i.e. for stars targeted by the LSS-GAC main and M31/M33 surveys. As shown by Yuan et al. (2015), the method yields an $E(B-V)$ precision of about 0.07 mag and works best for dwarf stars. $E(B-V)$ thus estimated is denoted 'EBV_PHOT' in the catalogues.

Considering that the star-pair method is model free, and often achieves a better precision compared to other methods, $E(B-V)$ yielded by the method ('EBV_SP') are generally adopted as the recommended values. Considering that there are few hot stars in the control sample used by the star-pair method, 'E(B-V)_MOD' is adopted as the recommended for stars hotter than $9000 \mathrm{~K}$. Since the SFD98 map is a two dimensional one, it provides an upper limit of $E(B-V)$ for reddening towards the individual Galactic stars. Based on this consideration, if the $E(B-V)$ value of a star deduced from the star-pair method or from the method based on model atmospheres excess the SFD98 value by $0.1 \mathrm{mag}(\sim 3 \sigma)$, the latter is adopted as the recommended value. The distribution of adopted $\mathrm{E}(\mathrm{B}-\mathrm{V})$ values for all stars in LSS-GAC DR2 are shown

\footnotetext{
3 http://classic.sdss.org/dr7/instruments/imager/index.html\#filters

4 http://www.ipac.caltech.edu/2mass/releases/second/doc/sec3_1b1.html
}
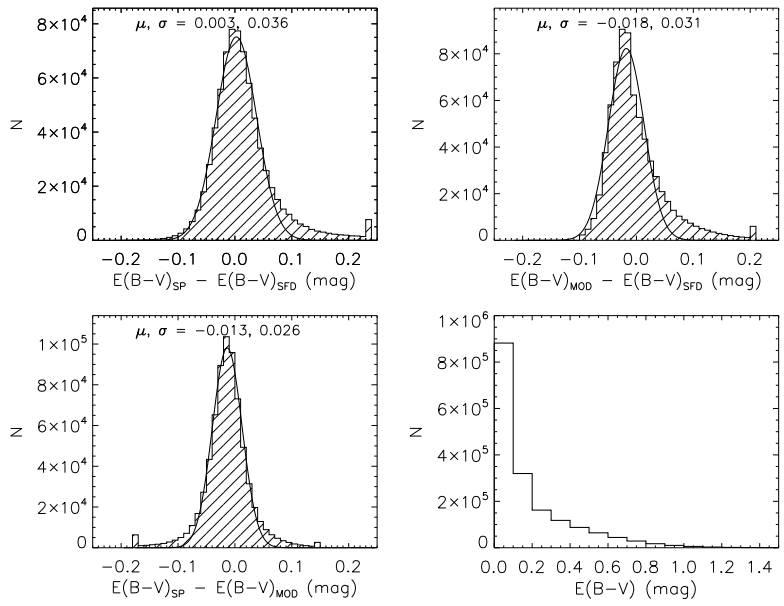

Figure 16. Distributions of differences of $E(B-V)$ estimated by different methods or from the SFD98 map except for the bottom-right panel where the distribution is for the adopted values of $E(B-V)$ for all stars in LSSGAC DR2. $E(B-V)_{\mathrm{SP}}$ and $E(B-V)_{\mathrm{MOD}}$ denote respectively values derived from the star-pair and stellar model atmosphere methods, whereas $E(B-V)_{\mathrm{SFD}}$ refers to values extracted from the 2-dimensional reddening map of Schlegel, Finkbeiner \& Davis (1998). The comparisons are for stars of $|b|>15^{\circ}$ and $E(B-V)_{\mathrm{SFD}}<0.3$ mag only.

in the bottom-right panel of Fig. 16. With $E(B-V)$ determined, values of interstellar extinction in the individual photometric bands can be easily computed using the extinction coefficients available from, for example, Yuan, Liu \& Xiang (2013).

\subsection{Distance}

Distance to individual stars are also estimated with a variety of methods. One is to infer from the absolute magnitudes derived directly from the LAMOST spectra with the KPCA method using the LAMOST-Hipparcos training set. In doing so, if photometric magnitudes of all $g, r$ and $K_{\mathrm{S}}$ bands have an error smaller than $0.1 \mathrm{mag}$, and are not saturated, then both $\mathrm{M}_{V}$ and $\mathrm{M}_{K_{\mathrm{s}}}$ are used to calculate a weighted-mean distance by taking the inverse squared magnitude errors as weights, otherwise only bands with good photometry are utilized to infer the distance. Generally, $K_{\mathrm{s}}$-band contributes a larger weight as it suffers from much less severe extinction than $V$-band. The $V$-band photometry is transformed from $g$ and $r$ magnitudes using the equation of Jester et al. (2005). Errors of absolute magnitudes, extinction and photometry are all incorporated in the estimation of distance errors. Note that it is found that the estimated $\mathrm{M}_{V}$ and $\mathrm{M}_{K_{\mathrm{s}}}$ are not independent quantities but largely correlated with each other, so that when estimating errors for the weighted-mean distance, we simply adopt the weightedmean distance errors in the individuals bands rather than infer from the reduced variance as done for independent measurements. This may have overestimated the distance errors to some extent. Distance estimated with this method is denoted as 'DIST_SP1' and 'DIST_SP2' in the value-added catalogues for results inferred from absolute magnitudes using 300 and 100 PCs, respectively.

Another method to estimate stellar distance is based on empirical relations between the absolute magnitudes and stellar atmospheric parameters constructed utilizing the MILES stars. A detailed description of the derivation of the empirical relations is presented in (Yuan et al. 2015). Here since the atmospheric parame- 

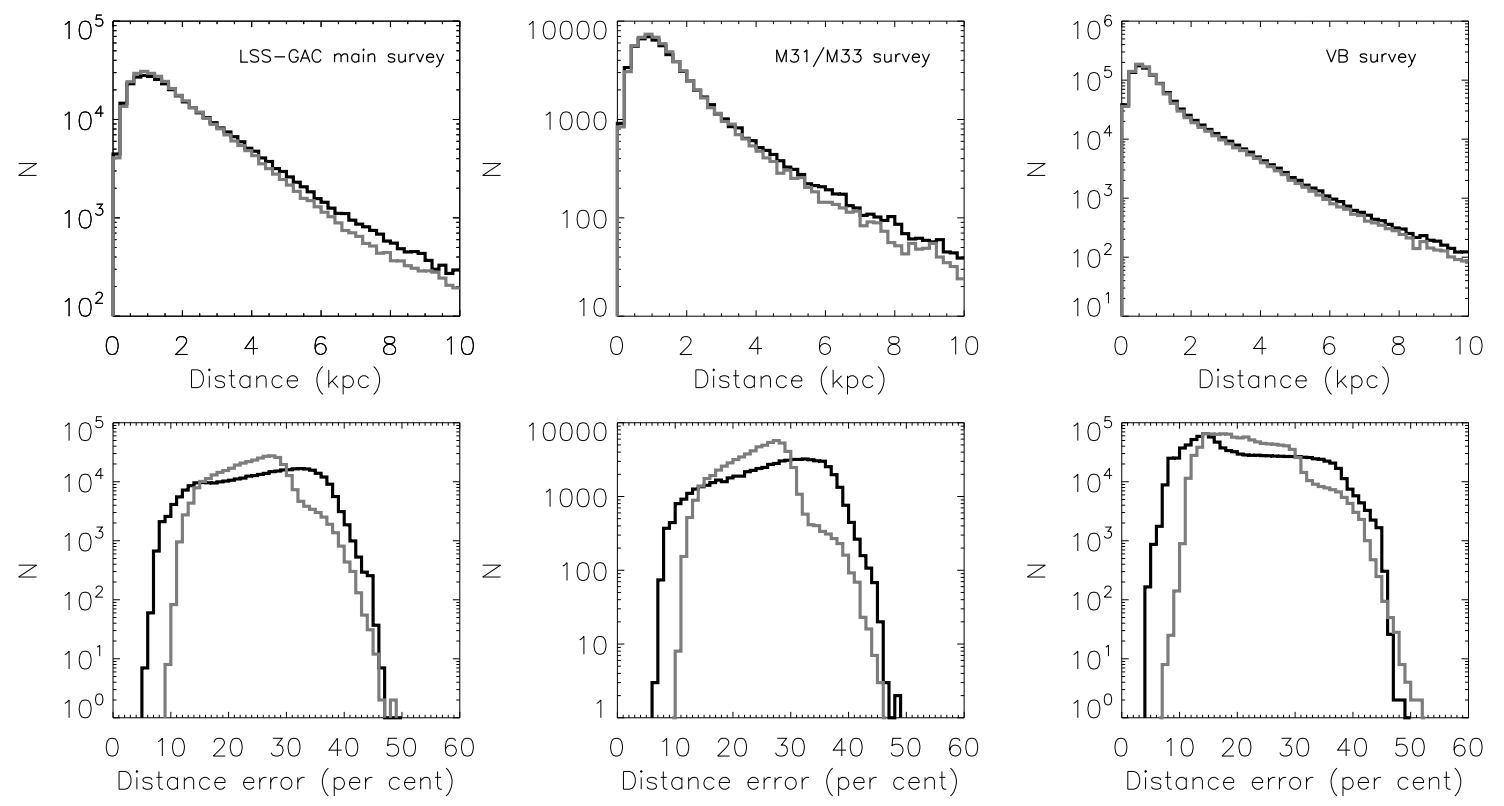

Figure 17. Distributions of distances and errors of stars targeted by the LSS-GAC main (left), M31/M33 (middle) and VB (right) surveys. Black and grey lines represent distances inferred from absolute magnitudes derived from LAMOST spectra with the KPCA method using 300 and 100 PCs, respectively.
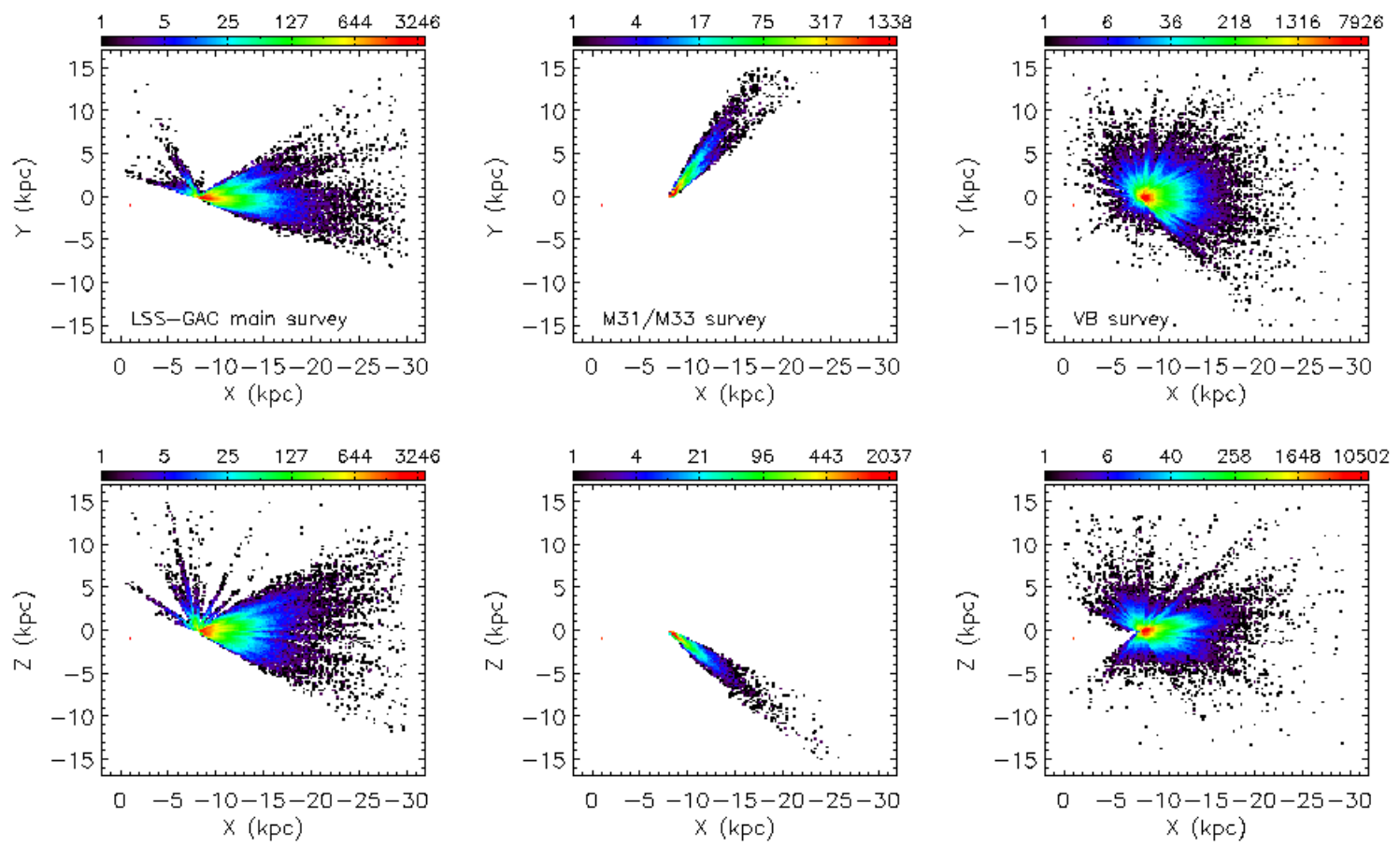

Figure 19. Colour-coded stellar density distributions in the Galactic $X-Y$ and $X-Z$ planes for stars targeted by the LSS-GAC main (left), M31/M33 (middle) and VB (right) surveys.

ters of MILES stars have been re-determined/calibrated (cf. §4.1), the relations are updated accordingly. The new relations have much smaller residuals - with dispersions of $g$ (and $r$ ) band absolute magnitudes of only about $0.08,0.13,0.15$ and 0.20 mag for OBA stars, FGK dwarfs, KM dwarfs and GKM giants, respectively. The corresponding residuals in the 2MASS infrared bands are about $0.15,0.26,0.06$ and 0.26 mag. The small residuals suggest that the method is robust. Errors of absolute magnitudes thus derived for the individual LSS-GAC stars are mainly contributed by uncertainties of the stellar atmospheric parameters. Here both the recommended atmospheric parameters and atmospheric parameters yielded by the weighted-mean algorithm are used to estimate absolute magnitudes. To obtain realistic error estimates of absolute magnitudes, we adopt a Monte-Carlo strategy to propagate the errors of stel- 


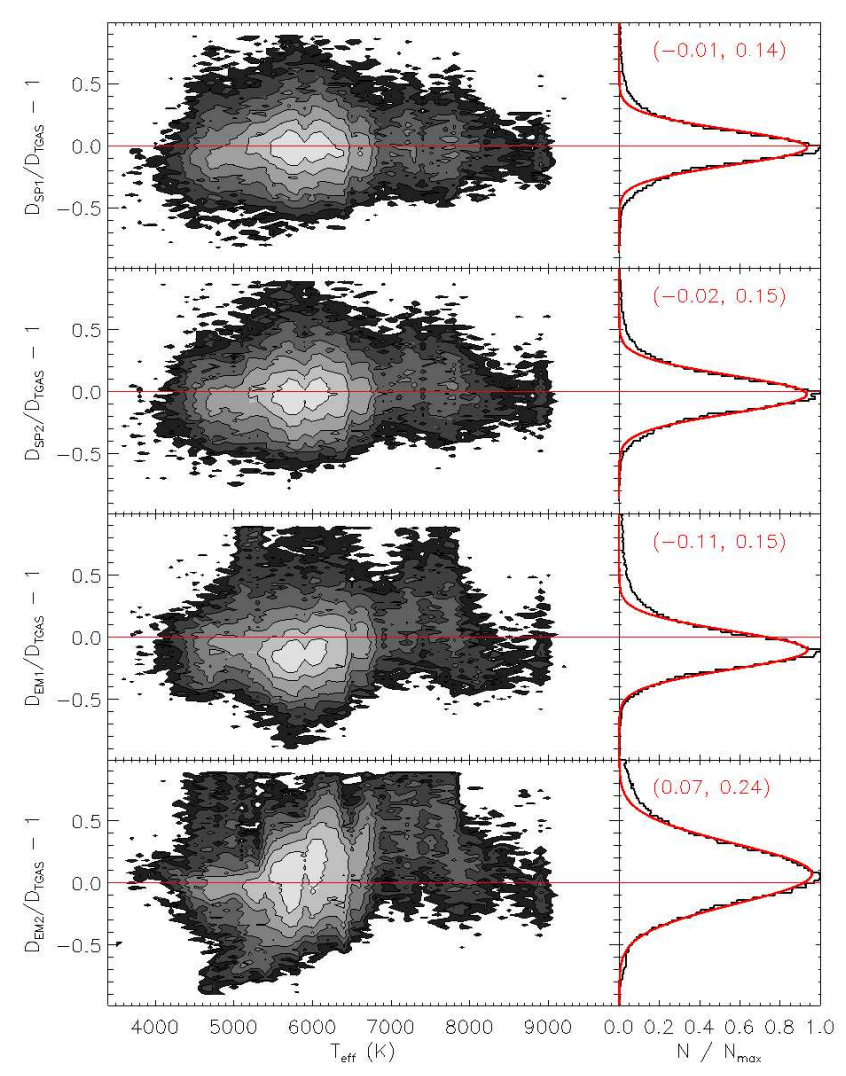

Figure 18. Percentage differences of distances between our estimates and those inferred from Gaia TGAS parallax as a function of the recommended $T_{\text {eff }}$ for a sample of 100,000 common stars. For up to low, the four panels show results for our distance estimates inferred from absolute magnitudes derived from LAMOST spectra using 300 PCs (first panel), 100 PCs (second panel) and from absolute magnitudes estimated using empirical relations utilizing the recommended parameters (third panel) and the weightedmean parameters (fourth panel). An error cut of 0.3 mag in the TGAS-based magnitudes is applied.

lar atmospheric parameters. The distance errors are then estimated taking into account contributions by errors in absolute magnitudes, extinction and photometry. Distances estimated with this method using the recommended parameters are denoted as 'DIST_EM1' in the value-added catalogues, and those using the weighted-mean atmospheric parameters are denoted as 'DIST_EM2'. In addition, stellar distances derived from photometry by Chen et al. (2014) via fitting stellar colour loci, if available, are also presented in the value-added catalogues, and are denoted by 'DIST_PHOT'.

Estimating distance using absolute magnitudes derived directly from the LAMOST spectra does not depend on stellar model atmospheres and stellar atmospheric parameters, and thus are expected to suffer from minimal systematics. Comparison with distances inferred from the Gaia TGAS parallaxes (Fig. 8) demonstrates that the overall systematic errors in our inferred distances are negligible, and the random errors can be as small as $\sim 10$ per cent given high enough spectral SNRs. Uncertainties in absolute magnitude thus distance estimates are sensitive to spectral SNRs. For a SNR of 10, the uncertainty increases to 30-40 per cent. Fig. 17 further shows that distance estimates from absolute magnitudes derived using both 300 and 100 PCs have essentially negligible (1-2 per cent) systematic errors at all $T_{\text {eff }}$ in the range of $5000-9000 \mathrm{~K}$.
Note that to incorporate stars in a wide temperature range for the comparison, especially for the high temperature end, here we have adopted an error cut of 0.3 mag for the TGAS-based magnitudes instead of $0.2 \mathrm{mag}$ as adopted for Fig. 8. As a result, the dispersion become 14 per cent, which is larger than 12 per cent shown in Fig. 8. While for stars cooler than $5000 \mathrm{~K}$, our distances seem to be underestimated by $5-10$ per cent. The figure shows also that distances yielded by the empirical relations using the recommended parameters are systematically underestimated by about 11 per cent, and the systematics show moderate dependence on $T_{\text {eff }}$. Such an underestimation is probably caused by either hidden systematic errors in the stellar atmospheric parameters, especially $\log g$, or by systematic errors in the empirical relations. Note that although the current parameter estimates achieved high precisions, potential systematic errors could be existed, which are probably a few tens Kelvin in $T_{\text {eff }}$, a few per cent to 0.1 dex in $\log g$ and a few per cent to $0.1 \mathrm{dex}$ in $[\mathrm{Fe} / \mathrm{H}]$. Potential systematic errors for metal-poor stars $([\mathrm{Fe} / \mathrm{H}]<-1.0$ dex $)$ are likely larger and should be treated cautiously. Distances yielded by the empirical relations using the weighted-mean parameters are systematically overestimated by 6 per cent, and there are strong patterns.

Distances inferred from absolute magnitudes directly determined from the LAMOST spectra using 300 PCs ('DIST_SP1') are currently adopted as the recommended values. It is emphasized that users should choose a proper set according to their own problems of interest. Fig. 17 plots the distributions of distances and error estimates for 'DIST_SP1' and 'DIST_SP2' for LSS-GAC DR2 stars of the main, M31/M33 and VB surveys, respectively. The distances peak at about $1 \mathrm{kpc}$, but with a few beyond $10 \mathrm{kpc}$. For the whole sample, 22, 5 and 2 per cent of the stars have a distance larger than 2,5 and $10 \mathrm{kpc}$, respectively. The quoted errors have a wide distribution from $\sim 7$ to $>40$ per cent. For 'DIST_SP1', about 11.5, 25.5, 63.0 and 98.8 per cent of the stars have a quoted error smaller than 15, 20,30 , and 40 per cent, respectively. The numbers are respectively 4.6, 22.9, 87.8 and 99.7 per cent for distances inferred from absolute magnitudes derived using 100 PCs. Finally, note that as mentioned in Section 4, there are about 18 per cent of stars that the KPCA method has failed to provide reliable estimates of absolute magnitudes due to low spectral SNRs. For those stars, the recommend distances are not available, while as a compromise one may use distance derived with the empirical relations utilizing the weighted-mean atmospheric parameters.

Fig. 19 plots the density distributions of LSS-GAC DR2 stars in the Galactic $X-Y$ and $X-Z$ planes for the main, M31/M33 and VB surveys, separately. Here the $X, Y$ and $Z$ are calcuated assuming $R_{\odot}=8.0 \mathrm{kpc}$ and $Z_{\odot}=0 \mathrm{kpc}$, and the recommended distances are used for the calculation. The figure shows that the main survey samples the outer disk well, while the VB survey samples the local disk very well. Some stars are located beyond $20 \mathrm{kpc}$ in the anti-centre direction and some are beyond $10 \mathrm{kpc}$ in the vertical direction.

\section{MULTI-BAND PHOTOMETRY, PROPER MOTION AND ORBITAL PARAMETERS}

Multi-band photometry from the far-UV to the mid-IR, including magnitudes in $F U V$ and $N U V$ bands from the Galaxy Evolution Explorer (GALEX; Martin et al. 2005), $g, r, i$ bands from the XSTPS-GAC, SDSS and APASS, $J, H, K_{\mathrm{s}}$ bands from the 2MASS and $w_{1}, w_{2}, w_{3}, w_{4}$ bands from the Wide - field Infrared Survey Explorer (WISE; Wright et al. 2010), are in- 
cluded in the value-added catalogues using with a 3 arc-second match radius for source cross-identifications.

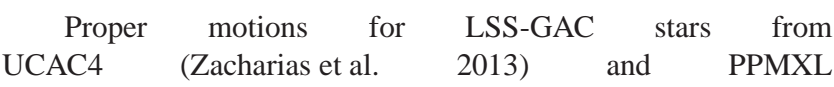
(Roeser, Demleitner \& Schilbach 2010), as well as those derived by combing the XSTPS-GAC and 2MASS catalogues (Yuan et al. 2015), are also included in the value-added catalogues.

Given celestial position, distance, radial velocity and proper motions, the stellar orbital parameters, including eccentricity, epicentre and pericentre radii, minimum and maximum vertical distances from the disk, are computed assuming a simple, axisymmetric Galactic potential as presented in Table 1 of Gardner \& Flynn (2010). The Sun is assumed to be located at $(\mathrm{X}, \mathrm{Y}, \mathrm{Z})=$ $(-8,0,0) \mathrm{kpc}$, and the local standard of rest (LSR) has values $\left(U_{\odot}, V_{\odot}, W_{\odot}\right)=(7.01,10.13,4.95) \mathrm{km} \mathrm{s}^{-1}$ (Huang et al. 2015b). When calculating the orbital parameters, systematic trends in proper motions with celestial positions are corrected for using the relations presented in Carlin et al. (2013) and in Huang et al. (2015b) for PPMXL and UCAC4 proper motions, respectively. Note that the systematics in proper motions have also been found to correlate with colour and magnitudes (e.g. Yuan et al. 2015; Sun et al. 2015). Such systematics have not been accounted for in the current treatment. Fig. 20 shows the eccentricity distribution of the sample stars.

\section{FORMAT OF THE VALUE-ADDED CATALOGUES}

Similar to LSS-GAC DR1, this second release of value-added catalogues are stored in three binary tables in FITS format. The filenames are 'lss-gac_dr2_GAC.fits', 'lss-gac_dr2_M31.fits', '1ssgac_dr2_VB.fits', corresponding to the LSS-GAC main, M31/M33 and VB surveys, respectively. The value-added catalogues include information of the LAMOST observations, stellar parameters and error estimates, interstellar reddening, distance, multi-band photometry, as well as proper motions and orbital parameters for $1,790,633$ observations of 1,393,844 unique stars that have a spec-

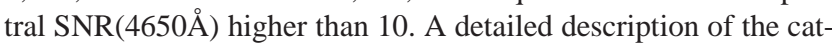
alogue content is presented in Table 5. Note that compared to LSSGAC DR1, more informations about the observations and stellar parameter estimates are provided for a better description of the data. This includes, for example, informations of Julian date at the start of the first exposure, airmass, lunar distance, as well as telescope altitude, and so on. Stellar type of the best-matching template is also provided, which should be helpful for identifying stars of special interest (cf. §4.5). The catalogues are accessible from http://lamost973.pku.edu.cn/site/node/4 along with a descriptive readme.txt file.

\section{SUMMARY}

The current work describes the second release (DR2) of valueadded catalogues of LSS-GAC, which contain observational information, multi-band photometry, radial velocity, stellar atmospheric parameters, elemental abundances, absolute magnitudes, extinction, distance, proper motions and orbital parameters for 1,790,633 LAMOST spectroscopic observations of 1,393,844 unique stars targeted by the LSS-GAC main, M31/M33 and VB surveys between September, 2011 and June, 2014 that have a spectral SNR

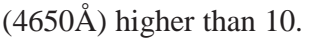

Spectra used to deliver LSS-GAC DR2 are processed at
Peking University with the LAMOST 2D pipeline and a spectral flux calibration pipeline specifically developed for LSS-GAC, as well as with the LAMOST Stellar Parameter Pipeline at Peking University (LSP3). Compared to LSS-GAC DR1, several improvements in stellar parameter determinations have been carried out, including (1) More than 260 new spectral templates observed with the YAO $2.4 \mathrm{~m}$ and NAOC $2.16 \mathrm{~m}$ telescopes have been added to the MILES library used by LSP3; (2) Atmospheric parameters of LSP3 template stars have been re-determined/calibrated to reduce the systematic and random errors; (3) Stellar atmospheric parameters $T_{\text {eff }}, \log g,[\mathrm{Fe} / \mathrm{H}],[\mathrm{M} / \mathrm{H}],[\alpha / \mathrm{Fe}],[\alpha / \mathrm{M}]$, absolute magnitudes $\mathrm{M}_{V}, \mathrm{M}_{K_{\mathrm{s}}}$ and elemental abundances $[\mathrm{C} / \mathrm{H}]$ and $[\mathrm{N} / \mathrm{H}]$ are deduced from LAMOST spectra with a KPCA-based multivariate regression method. The method yields robust results for spectra of good SNRs $(>50)$ - estimates of $\log g$ are found to be accurate to 0.1 dex for giants and late-type dwarfs, and estimates of $\mathrm{M}_{V}$ and $\mathrm{M}_{K_{\mathrm{s}}}$ are accurate to $0.3 \mathrm{mag}$, corresponding to a distance accuracy of about 15 per cent. Distances inferred from absolute magnitudes determined directly from LAMOST spectra using the LAMOSTHipparcos training set are free from stellar model atmospheres and stellar atmospheric parameters and are thus expected to have minimal systematics. Estimates of $[\mathrm{M} / \mathrm{H}],[\mathrm{Fe} / \mathrm{H}],[\mathrm{C} / \mathrm{H}]$ and $[\mathrm{N} / \mathrm{H}]$ for giant stars are shown to have a precision better than $0.1 \mathrm{dex}$, and those of $[\alpha / \mathrm{Fe}]$ and $[\alpha / \mathrm{M}]$ better than $0.05 \mathrm{dex}$; (4) Estimates of $[\alpha / \mathrm{Fe}]$ by template matching with the KURUCZ synthetic spectra are also provided for both dwarfs and giants; (5) More descriptive critical flags and information about the observations and stellar parameter determination are provided to help better understand the data and find, for instance, stars of special interest, or to mask stars with poorly determined parameters; (6) Realistic errors are estimated for atmospheric parameters, distance and other inferred quantities (e.g. 3-dimensional positions and velocities). Parameter errors are estimated in detail by taking in account of contributions from all identified sources, including spectral noise, inadequacy of the methods and templates adopted for the analysis, and errors propagated from other relevant parameters. Errors are assigned to the individual determinations for each star based on the spectral SNR and stellar atmospheric parameters.

Besides the large number of stars, the data sets cover a large and almost contiguous volume of the Galactic disk and halo, and have a relatively simple target selection function. They are thus expected to serve as a unique and valuable asset to study the structure and evolution of the Galaxy, especially the disk and the solar neigbourhood. In addition, about 28 per cent measurements in the value-added catalogues are duplicate results of the same targets. The data sets are therefore also useful for time-domain spectroscopic studies.

Acknowledgments This work is supported by National Key Basic Research Program of China 2014CB845700 and Joint Funds of the National Natural Science Foundation of China (Grant No. U1531244 and U1331120). Guoshoujing Telescope (the Large Sky Area Multi-Object Fiber Spectroscopic Telescope LAMOST) is a National Major Scientific Project built by the Chinese Academy of Sciences. Funding for the project has been provided by the National Development and Reform Commission. LAMOST is operated and managed by the National Astronomical Observatories, Chinese Academy of Sciences. We acknowledge the support of the staff of the Xinglong $2.16 \mathrm{~m}$ telescope and the Lijiang $2.4 \mathrm{~m}$ telescope. This work was partially Supported by the Open Project Program of the Key Laboratory of Optical Astronomy, National 

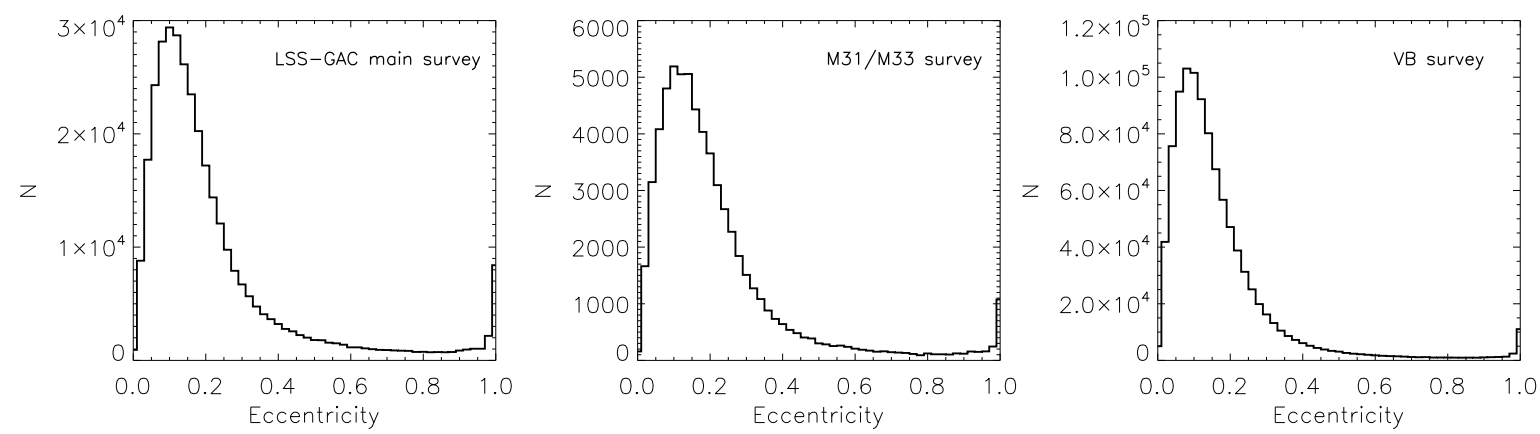

Figure 20. Distributions of orbital eccentricities for stars targeted by the LSS-GAC main (left), M31/M33 (middle) and the VB (right) surveys.

Astronomical Observatories, Chinese Academy of Sciences. H.-B. Yuan is supported by NSFC grant No. 11443006, No. 11603002 and Beijing Normal University grant No. 310232102. This work has made use of data products from the Galaxy Evolution Explorer (GALEX), Xuyi Schmidt Telescope Photometric Survey of the Galactic Anticentre (XSTPS-GAC), Sloan Digital Sky Survey (SDSS), AAVSO Photometric All-Sky Survey (APASS), Two Micron All Sky Survey (2MASS), Wide-field Infrared Survey Explorer (WISE), NASA/IPAC Infrared Science Archive and the cross-match service provided by CDS, Strasbourg. We acknowledge the valuable suggestions from the anonymous referee.

\section{REFERENCES}

Aihara H. et al., 2011, ApJS, 193, 29

Anderson E., Francis C., 2012, Astronomy Letters, 38, 331

Carlin J. L. et al., 2013, ApJL, 777, L5

Carlin J. L. et al., 2015, AJ, 150, 4

Castelli F., Kurucz R. L., 2004, ArXiv Astrophysics e-prints

Cenarro A. J. et al., 2007, MNRAS, 374, 664

Chen B.-Q. et al., 2014, MNRAS, 443, 1192

Cui X.-Q. et al., 2012, Research in Astronomy and Astrophysics, 12, 1197

Deng L.-C. et al., 2012, Research in Astronomy and Astrophysics, 12,735

Doi M. et al., 2010, AJ, 139, 1628

Dotter A., Chaboyer B., Jevremović D., Kostov V., Baron E., Ferguson J. W., 2008, ApJS, 178, 89

Falcón-Barroso J., Sánchez-Blázquez P., Vazdekis A., Ricciardelli E., Cardiel N., Cenarro A. J., Gorgas J., Peletier R. F., 2011, A\&A, 532, A95

Gao H. et al., 2015, Research in Astronomy and Astrophysics, 15, 2204

García Pérez A. E. et al., 2015, ArXiv e-prints

Gardner E., Flynn C., 2010, MNRAS, 405, 545

Gray R. O., 1999, SPECTRUM: A stellar spectral synthesis program. Astrophysics Source Code Library

Gunn J. E. et al., 1998, AJ, 116, 3040

Ho A. Y. Q. et al., 2016, ArXiv e-prints

Holtzman J. A. et al., 2015, AJ, 150, 148

Huang Y., Liu X.-W., Yuan H.-B., Xiang M.-S., Chen B.-Q., Zhang H.-W., 2015a, MNRAS, 454, 2863

Huang Y., Liu X.-W., Yuan H.-B., Xiang M.-S., Huo Z.-Y., Chen

B.-Q., Zhang Y., Hou Y.-H., 2015b, MNRAS, 449, 162

Huang Y. et al., 2015c, Research in Astronomy and Astrophysics, 15,1240
Jester S. et al., 2005, AJ, 130, 873

Jofré P. et al., 2015, A\&A, 582, A81

Lee Y. S. et al., 2011, AJ, 141, 90

Lee Y. S. et al., 2015, AJ, 150, 187

Li J. et al., 2016, Research in Astronomy and Astrophysics, 16, 010

Lindegren L. et al., 2016, ArXiv e-prints

Liu C. et al., 2015, ApJ, 807, 4

Liu X.-W. et al., 2014, in IAU Symposium, Vol. 298, IAU Symposium, Feltzing S., Zhao G., Walton N. A., Whitelock P., eds., pp. 310-321

Luo A.-L. et al., 2012, Research in Astronomy and Astrophysics, 12,1243

Luo A.-L. et al., 2015, Research in Astronomy and Astrophysics, 15,1095

Majewski S. R., Wilson J. C., Hearty F., Schiavon R. R., Skrutskie M. F., 2010, in IAU Symposium, Vol. 265, IAU Symposium, Cunha K., Spite M., Barbuy B., eds., pp. 480-481

Martin D. C. et al., 2005, ApJL, 619, L1

Milligan S., Cranton B. W., Skrutskie M. F., 1996, in Proc. SPIE, Vol. 2863, Current Developments in Optical Design and Engineering VI, Fischer R. E., Smith W. J., eds., pp. 2-13

Munari U. et al., 2014, AJ, 148, 81

Munari U., Sordo R., Castelli F., Zwitter T., 2005, A\&A, 442, 1127

Perryman M. A. C. et al., 2001, A\&A, 369, 339

Perryman M. A. C. et al., 1997, A\&A, 323, L49

Rebassa-Mansergas A. et al., 2015, MNRAS, 450, 743

Ren J.-J. et al., 2016, Research in Astronomy and Astrophysics, 16, 009

Roeser S., Demleitner M., Schilbach E., 2010, AJ, 139, 2440

Rosenfield P., Marigo P., Girardi L., Dalcanton J. J., Bressan A., Williams B. F., Dolphin A., 2016, ApJ, 822, 73

Sánchez-Blázquez P. et al., 2006, MNRAS, 371, 703

Schlegel D. J., Finkbeiner D. P., Davis M., 1998, ApJ, 500, 525

Skrutskie M. F. et al., 2006, AJ, 131, 1163

Soubiran C., Le Campion J.-F., Cayrel de Strobel G., Caillo A., 2010, A\&A, 515, A111

Sun N.-C. et al., 2015, Research in Astronomy and Astrophysics, 15,1342

Wang J. et al., 2016a, MNRAS, 456, 672

Wang L. et al., 2016b, AJ, 152, 6

Wenger M. et al., 2000, A\&AS, 143, 9

Wright E. L. et al., 2010, AJ, 140, 1868

Wu Y., Du B., Luo A., Zhao Y., Yuan H., 2014, in IAU Symposium, Vol. 306, Statistical Challenges in 21st Century Cosmol- 
ogy, Heavens A., Starck J.-L., Krone-Martins A., eds., pp. 340342

Wu Y. et al., 2011, Research in Astronomy and Astrophysics, 11, 924

Xiang M.-S. et al., 2017, MNRAS, 464, 3657

Xiang M. S. et al., 2015a, MNRAS, 448, 822

Xiang M.-S. et al., 2015b, Research in Astronomy and Astrophysics, 15, 1209

Xiang M. S. et al., 2015c, MNRAS, 448, 90

York D. G. et al., 2000, AJ, 120, 1579

Yuan H.-B. et al., 2015, MNRAS, 448, 855

Yuan H. B., Liu X. W., Xiang M. S., 2013, MNRAS, 430, 2188

Zacharias N., Finch C. T., Girard T. M., Henden A., Bartlett J. L., Monet D. G., Zacharias M. I., 2013, AJ, 145, 44

Zhang H.-H., Liu X.-W., Yuan H.-B., Zhao H.-B., Yao J.-S., Zhang H.-W., Xiang M.-S., 2013, Research in Astronomy and Astrophysics, 13, 490

Zhang H.-H., Liu X.-W., Yuan H.-B., Zhao H.-B., Yao J.-S., Zhang H.-W., Xiang M.-S., Huang Y., 2014, Research in Astronomy and Astrophysics, 14, 456

Zhao G., Zhao Y.-H., Chu Y.-Q., Jing Y.-P., Deng L.-C., 2012, Research in Astronomy and Astrophysics, 12, 723 
Table 5. Description of the second release of LSS-GAC value-added catalogues.

\begin{tabular}{|c|c|c|}
\hline Col. & Name & Description \\
\hline 1 & spec_id & LAMOST unique spectral ID, in format of date-plateid-spectrographid-fibreid \\
\hline 2 & date & Date of observation \\
\hline 3 & plate & LAMOST plate ID, not necessarily unique \\
\hline 4 & sp_id & LAMOST spectrograph ID, ranging from 1 to 16 \\
\hline 5 & fiber_id & LAMOST fibre ID for a given spectrograph, ranging from 1 to 250 \\
\hline 6 & objid & Object ID in the input catalogues \\
\hline 7 & objtype & Initial object type from the survey input catalogues \\
\hline 8 & ra & Right ascension of $\mathbf{J} 2000.0$ (०) \\
\hline 9 & dec & Declination of J2000.0 (०) \\
\hline 10 & 1 & Galactic longitude (o) \\
\hline 11 & $\mathrm{~b}$ & Galactic latitude (o) \\
\hline 12 & jd & Julian days at the middle time of the first exposure \\
\hline 13 & moonphase & Moon phase at the middle time of the first exposure \\
\hline 14 & monodis & Moon distance at the middle time of the first exposure \\
\hline 15 & airmass & Airmass at the middle time of the first exposure \\
\hline 16 & alt & Altitude (in degrees) of the telescope at the middle time of the first exposure \\
\hline 17 & $\mathrm{az}$ & Azimuth angle (in degrees) of the telescope at the middle time of the first exposure \\
\hline 18 & ha & Hour angle (in degrees) of the telescope at the middle time of the first exposure \\
\hline 19 & badfiber & Is it a bad fibre? (yes -1, no -0$)$ \\
\hline 20 & satflag & Is the spectrum saturated? (yes -1, no -0 ) \\
\hline 21 & brightflag & Are there bright stars $(\mathrm{snr}-\mathrm{b}>300$ ) in the nearby 5 fibres (yes -1 , no -0 ) \\
\hline 22 & brightsnr & Largest snr_b in the nearby 5 fibres \\
\hline 23 & object_sky_ratio & Object to sky flux ratio \\
\hline 24 & snr_b & $\mathrm{S} / \mathrm{N}(4650 \AA ̊)$ per pixel \\
\hline 25 & snr_r & $\mathrm{S} / \mathrm{N}(7450 \AA)$ per pixel \\
\hline 26 & uqflag & $\begin{array}{l}\text { Uniqueness flag. If the target has been observed } \mathrm{n} \text { times, then uqflag runs from } 1 \text { to } \mathrm{n} \text {, with } 1 \text { denoting } \\
\text { the spectrum of the highest snr_b }\end{array}$ \\
\hline 27 & $\mathrm{vr}$ & Radial velocity yielded by LSP3, after corrected for a systematic offset of $-3.1 \mathrm{~km} \mathrm{~s}^{-1}\left(\mathrm{~km} \mathrm{~s}^{-1}\right)$ \\
\hline 28 & err_vr & Error of radial velocity yielded by LSP3 $\left(\mathrm{km} \mathrm{s}^{-1}\right)$ \\
\hline 29 & teff & Recommended $T_{\text {eff }}(\mathrm{K})$ \\
\hline 30 & err_teff & Error of the recommended $T_{\text {eff }}$ \\
\hline 31 & teff_from & Which method is the recommended $T_{\text {eff }}$ adopted \\
\hline 32 & $\log g$ & Recommended $\log g\left(\mathrm{~cm} \mathrm{~s}^{-2}\right)$ \\
\hline 33 & err_logg & Error of the recommended $\log g$ \\
\hline 34 & logg_from & Which method is the recommended $\log g$ adopted \\
\hline 35 & $\mathrm{mv}$ & Recommended absolute magnitude in $V$-band \\
\hline 36 & err_mv & Error of $\mathrm{M}_{V}$ \\
\hline 37 & mv_from & Which method is the recommended $\mathrm{M}_{V}$ adopted \\
\hline 38 & $\mathrm{mk}$ & Recommended absolute magnitude in $K_{\mathrm{s}}$-band \\
\hline 39 & err_mk & Error of $\mathrm{M}_{K_{\mathrm{s}}}$ \\
\hline 40 & mk_from & Which method is the recommended $\mathrm{M}_{K_{\mathrm{s}}}$ adopted \\
\hline 41 & feh & Recommended $[\mathrm{Fe} / \mathrm{H}](\mathrm{dex})$ \\
\hline 42 & err_feh & Error of the recommended $[\mathrm{Fe} / \mathrm{H}]$ \\
\hline 43 & feh_from & Which method is the recommended $[\mathrm{Fe} / \mathrm{H}]$ adopted \\
\hline 44 & afe & Recommended $[\alpha / \mathrm{Fe}](\mathrm{dex})$ \\
\hline 45 & err_afe & Error of the recommended $[\alpha / \mathrm{Fe}]$ \\
\hline 46 & afe_from & Which method is the recommended $[\alpha / \mathrm{Fe}]$ adopted \\
\hline 47 & am & $\alpha$-element to metal abundance ratio $[\alpha / \mathrm{M}]$ estimated by training the LAMOST-APOGEE stars (dex) \\
\hline 48 & err_am & Error of $[\alpha / \mathrm{M}]$ \\
\hline 49 & $\mathrm{mh}$ & Metal abundance $[\mathrm{M} / \mathrm{H}]$ estimated by training the LAMOST-APOGEE stars (dex) \\
\hline 50 & err_mh & Error of $[\mathrm{M} / \mathrm{H}]$ \\
\hline 51 & $\mathrm{ch}$ & Carbon abundance $[\mathrm{C} / \mathrm{H}]$ estimated by training the LAMOST-APOGEE stars (dex) \\
\hline 52 & err_mh & Error of $[\mathrm{C} / \mathrm{H}]$ \\
\hline 53 & $\mathrm{nh}$ & Nitrogen abundance $[\mathrm{N} / \mathrm{H}]$ estimated by training the LAMOST-APOGEE stars (dex) \\
\hline 54 & err_nh & Error of $[\mathrm{N} / \mathrm{H}]$ \\
\hline 55 & ebv & Recommended $E(B-V)$ \\
\hline 56 & dist & Recommended distance (pc) \\
\hline 57 & err_dist & Error of the recommended distance \\
\hline 58 & dist_from & Which method is the recommended distance adopted \\
\hline 59 & vr_peak_corr_coeff & Peak correlation coefficient for radial velocity estimation \\
\hline 60 & vr_flag & $\begin{array}{l}\text { Flag to describe value of 'vr_peak_corr_coeff', } n \text { means the vr_peak_corr_coeff } \\
\text { is smaller than the median value of the sample stars with the same snr_b by } n \times \text { MAD }\end{array}$ \\
\hline
\end{tabular}


Table 6. - continued

\begin{tabular}{|c|c|c|}
\hline Col. & Name & Description \\
\hline 61 & teff1 & $T_{\text {eff }}$ estimated with the weighted-mean algorithm (K) \\
\hline 62 & err_teff 1 & Error of teff1 \\
\hline 63 & teff2 & $T_{\text {eff }}$ estimated with the KPCA method (K) \\
\hline 64 & err_teff2 & Error of teff2 \\
\hline 65 & $\operatorname{logg} 1$ & $\log g$ estimated with the weighted-mean algorithm $\left(\mathrm{cm} \mathrm{s}^{-2}\right)$ \\
\hline 66 & err_logg 1 & Error of $\operatorname{logg} 1$ \\
\hline 67 & $\operatorname{logg} 2$ & Log $g$ estimated with the KPCA method using MILES stars as training set $\left(\mathrm{cm} \mathrm{s}^{-2}\right)$ \\
\hline 68 & err_logg2 & Error of $\operatorname{logg} 2$ \\
\hline 69 & $\log 3$ & Log $g$ estimated with the KPCA method using LAMOST-Kepler stars as training set $\left(\mathrm{cm} \mathrm{s}^{-2}\right)$ \\
\hline 70 & err_logg3 & Error of $\operatorname{logg} 3$ \\
\hline 71 & mv1 & $\mathrm{M}_{V}$ estimated with the KPCA method taking LAMOST-Hipparcos stars as training set using 300 PCs (mag) \\
\hline 72 & err_mv1 & Error of mv1 \\
\hline 73 & $\operatorname{mv} 2$ & $\mathrm{M}_{V}$ estimated with the KPCA method taking LAMOST-Hipparcos stars as training set using 100 PCs (mag) \\
\hline 74 & err_mv2 & Error of mv2 \\
\hline 75 & $\mathrm{mk} 1$ & $\mathrm{M}_{K_{\mathrm{s}}}$ estimated with the KPCA method taking LAMOST-Hipparcos stars as training set using 300 PCs (mag) \\
\hline 76 & err_mk1 & Error of $\mathrm{mk} 1$ \\
\hline 77 & $\mathrm{mk} 2$ & $\mathrm{M}_{K_{\mathrm{s}}}$ estimated with the KPCA method taking LAMOST-Hipparcos stars as training set using 100 PCs (mag) \\
\hline 78 & err_mk2 & Error of $m k 2$ \\
\hline 79 & feh1 & {$[\mathrm{Fe} / \mathrm{H}]$ estimated with the LSP3 weighted mean algorithm (dex) } \\
\hline 80 & err_feh1 & Error of feh1 \\
\hline 81 & feh2 & {$[\mathrm{Fe} / \mathrm{H}]$ estimated with the LSP3 KPCA method by training the MILES stars (dex) } \\
\hline 82 & err_feh2 & Error of feh2 \\
\hline 83 & feh3 & {$[\mathrm{Fe} / \mathrm{H}]$ estimated with the LSP3 KPCA method by training the LAMOST-APOGEE stars (dex) } \\
\hline 84 & err_feh3 & Error of feh3 \\
\hline 85 & afe 1 & $\begin{array}{l}\text { [ } \alpha / \mathrm{Fe}] \text { estimated by template matching with synthetic spectra using spectral segments } \\
\text { of } 3900-3980 \AA, 4400-4600 \AA \text { and } 5000-5300 \AA \text { (dex) }\end{array}$ \\
\hline 86 & err_afe1 & Error of afe 1 \\
\hline 87 & afe 2 & $\begin{array}{l}\text { [ } \alpha / \mathrm{Fe}] \text { estimated by template matching with synthetic spectra using spectral segments } \\
\text { of } 4400-4600 \AA \text { and } 5000-5300 \AA(\mathrm{dex})\end{array}$ \\
\hline 88 & err_afe2 & Error of afe 2 \\
\hline 89 & afe 3 & {$[\alpha / \mathrm{Fe}]$ estimated with the LSP3 KPCA method using the LAMOST-APOGEE training set (dex) } \\
\hline 90 & err_afe3 & Error of afe 3 \\
\hline 91 & besttemp_chi2 & Name of the best-matching template star with $\chi^{2}$ algorithm \\
\hline 92 & besttemp_corr & Name of the best-matching template star with correlation algorithm \\
\hline 93 & typeflag_chi2 & Stellar types of the best-matching template star with $\chi^{2}$ algorithm \\
\hline 94 & typeflag_corr & Stellar types of the best-matching template star with correlation algorithm \\
\hline 95 & min_chi2 & Minimum $\chi^{2}$ yielded by LSP3 from template matching with the MILES library \\
\hline 96 & chi2_flag & $\begin{array}{l}\text { The 'min_chi2' is smaller than the median value of the sample stars in the same SNR and } \\
\text { atmospheric parameter bin by } n \times \text { MAD }\end{array}$ \\
\hline 97 & dg_miles_tm & $\begin{array}{l}\text { Maximum value of the kernel function for estimation of } T_{\text {eff }} \text { and }[\mathrm{Fe} / \mathrm{H}] \text { with KPCA method } \\
\text { via training the MILES stars }\end{array}$ \\
\hline 98 & dg_miles_g & $\begin{array}{l}\text { Maximum value of the kernel function for estimation of } \log g \text { with KPCA method via training } \\
\text { the MILES stars }\end{array}$ \\
\hline 99 & dg_lm_kep & $\begin{array}{l}\text { Maximum value of the kernel function for estimation of } \log g \text { with KPCA method via training } \\
\text { the LAMOST-Kepler stars }\end{array}$ \\
\hline 100 & dg_lm_hip & $\begin{array}{l}\text { Maximum value of the kernel function for estimation of absolute magnitudes with KPCA method } \\
\text { via training the LAMOST-Hipparcos stars }\end{array}$ \\
\hline 101 & dg_lm_apo & $\begin{array}{l}\text { Maximum value of the kernel function for estimation of abundances with KPCA method via training } \\
\text { the LAMOST-APOGEE stars }\end{array}$ \\
\hline 102 & fuv & GALEX FUV band magnitude (mag) \\
\hline 103 & err_fuv & Error of fuv \\
\hline 104 & nuv & GALEX NUV band magnitude (mag) \\
\hline 105 & err_nuv & Error of nuv \\
\hline 106 & $\mathrm{~g}$ & XSTPS-GAC g-band magnitude (mag) \\
\hline 107 & err_g & Error of $\mathrm{g}$ \\
\hline 108 & $\mathrm{r}^{-0}$ & XSTPS-GAC r-band magnitude (mag) \\
\hline 109 & err_r & Error of $\mathrm{r}$ \\
\hline 110 & $\mathrm{i}^{-}$ & XSTPS-GAC i-band magnitude (mag) \\
\hline 111 & err_i & Error of $\mathrm{i}$ \\
\hline 112 & $\mathrm{~J}$ & 2MASS J-band magnitude (mag) \\
\hline 113 & err_J & Error of J \\
\hline 114 & $\mathrm{H}$ & 2MASS H-band magnitude (mag) \\
\hline 115 & err_H & Error of $\mathrm{H}$ \\
\hline 116 & Ks & 2MASS Ks-band magnitude (mag) \\
\hline 117 & err_Ks & Error of Ks \\
\hline
\end{tabular}


Table 7. - continued

\begin{tabular}{|c|c|c|}
\hline Col. & Name & Description \\
\hline 118 & w1 & WISE W1-band magnitude (mag) \\
\hline 119 & err_w1 & Error of w1 \\
\hline 120 & w2 & WISE W2-band magnitude (mag) \\
\hline 121 & err_w2 & Error of w2 \\
\hline 122 & w3 & WISE W3-band magnitude (mag) \\
\hline 123 & err_w3 & Error of w3 \\
\hline 124 & w4 & WISE W4-band magnitude (mag) \\
\hline 125 & err_w4 & Error of w4 \\
\hline 126 & ph_qual_2mass & 2MASS photometric quality flag \\
\hline 127 & ph_qual_wise & WISE photometric quality flag \\
\hline 128 & var_flag_wise & WISE variability flag \\
\hline 129 & ext_flag_wise & WISE extended source flag \\
\hline 130 & cc_flag_wise & WISE contamination and confusion flag \\
\hline 131 & ebv_sfd & $E(B-V)$ from the SFD extinction map (mag) \\
\hline 132 & ebv_sp & $E(B-V)$ derived from the star pair method (mag) \\
\hline 133 & err_ebv_sp & Error of ebv_sp \\
\hline 134 & ebv_mod & $E(B-V)$ derived by comparing the observed and synthetic model atmosphere colours (mag) \\
\hline 135 & ebv_phot & $E(B-V)$ derived by fitting multiband photometry to the empirical stellar loci (Chen et al. 2014) (mag) \\
\hline 136 & dist_em1 & $\begin{array}{l}\text { Distance derived using the empirical relations of absolute magnitudes as a function of stellar atmospheric } \\
\text { parameters as yielded by the MILES stars using the recommended atmospheric parameters }\end{array}$ \\
\hline 137 & err_dist_em1 & Error of dist_em1 \\
\hline 138 & dist_em2 & $\begin{array}{l}\text { Distance derived using the empirical relations of absolute magnitudes as a function of stellar atmospheric } \\
\text { parameters as yielded by the MILES stars using the weighted-mean atmospheric parameters }\end{array}$ \\
\hline 139 & err_dist_em2 & Error of dist_em2 \\
\hline 140 & dist_sp1 & Distance inferred from absolute magnitudes estimated with KPCA method using 300 PCs (pc) \\
\hline 141 & err_dist_sp1 & Error of dist_sp1 \\
\hline 142 & dist_sp2 & Distance inferred from absolute magnitudes estimated with KPCA method using 100 PCs (pc) \\
\hline 143 & err_dist_sp2 & Error of dist_sp2 \\
\hline 144 & dist_phot & Distance derived from the photometric parallax method (Chen et al. 2014) (pc) \\
\hline 145 & err_dist_phot & Error of dist_phot \\
\hline 146 & pmra_ppmxl & Proper motion in RA from the PPMXL catalogues $\left(\right.$ mas $\mathrm{yr}^{-1}$ ) \\
\hline 147 & epmra_ppmxl & Error of pmra_ppmxl \\
\hline 148 & pmdec_ppmxl & Proper motion in Dec. from the PPMXL catalogues (mas $\mathrm{yr}^{-1}$ ) \\
\hline 149 & epmdec_ppmxl & Error of pmdec_ppmxl \\
\hline 150 & pmra_ucac4 & Proper motion in RA from the UCAC4 catalogues ( mas $\mathrm{yr}^{-1}$ ) \\
\hline 151 & epmra_ucac4 & Error of pmra_ucac4 \\
\hline 152 & pmdec_ucac4 & Proper motion in Dec. from the UCAC4 catalogues ( mas $r^{-1}$ ) \\
\hline 153 & epmdec_ucac4 & Error of pmdec_ucac4 \\
\hline 154 & pmra_xuyi2mass & Proper motion in RA derived by comparing the XSTPS-GAC and 2MASS positions ( mas $\mathrm{yr}^{-1}$ ) \\
\hline 155 & pmdec_xuyi2mass & Proper motion in Dec. derived by comparing the XSTPS-GAC and 2MASS positions ( mas $\mathrm{yr}^{-1}$ ) \\
\hline 156 & $\mathrm{x}$ & $\mathrm{x}$ coordinate in a Galactocentric Cartesian reference system, positive towards the Galactic Centre $(\mathrm{kpc})$ \\
\hline 157 & err_x & Error of $x$ \\
\hline 158 & y & y coordinate in a Galactocentric Cartesian reference system, positive in the direction of disk rotation $(\mathrm{kpc})$ \\
\hline 159 & err_y & Error of y \\
\hline 160 & $\mathrm{z}$ & $\mathrm{z}$ coordinate in a Galactocentric Cartesian reference system, positive towards the North Galactic Pole (kpc) \\
\hline 161 & err_z & Error of $\mathrm{z}$ \\
\hline 162 & $\mathrm{u}[2]$ & $\begin{array}{l}\text { Galactic space velocities in the } \mathrm{x} \text { direction computed from dist_em and the corrected PPMXL and UCAC4 } \\
\text { proper motions, respectively, positive towards the Galactic Centre }\left(\mathrm{km} \mathrm{s}^{-1}\right)\end{array}$ \\
\hline 163 & err_u[2] & Error of $\mathrm{u}[2]$ \\
\hline 164 & $\mathrm{v}[2]$ & $\begin{array}{l}\text { Galactic space velocities in the y direction computed from dist_em and the corrected PPMXL and UCAC4 } \\
\text { proper motions, respectively, positive in the direction of Galactic rotation }\left(\mathrm{km} \mathrm{s}^{-1}\right)\end{array}$ \\
\hline 165 & err_v[2] & Error of $v[2]$ \\
\hline 166 & $\mathrm{w}[2]$ & $\begin{array}{l}\text { Galactic space velocities in the } \mathrm{z} \text { direction computed from dist_em and the corrected PPMXL and UCAC4 } \\
\text { proper motions, respectively, positive towards the North Galactic Pole }\left(\mathrm{km} \mathrm{s}^{-1}\right)\end{array}$ \\
\hline
\end{tabular}


Table 8. - continued

\begin{tabular}{|c|c|c|}
\hline Col. & Name & Description \\
\hline 167 & err_w[2] & Error of $w[2]$ \\
\hline 168 & v_r $[2]$ & $\begin{array}{l}\text { Galactic space velocities in the } \mathrm{r} \text { direction in a Galactocentric cylindrical polar coordinate system, } \\
\text { computed from dist_em and the corrected PPMXL and UCAC4 proper motions, respectively }\left(\mathrm{km} \mathrm{s}^{-1}\right)\end{array}$ \\
\hline 169 & err_v_r[2] & Error of $v_{-} r[2]$ \\
\hline 170 & v_phi[2] & $\begin{array}{l}\text { Galactic space velocities in the } \Phi \text { direction in a Galactocentric cylindrical polar coordinate system, } \\
\text { computed from dist_em and the corrected PPMXL and UCAC4 proper motions, respectively, } \\
\text { positive in the direction of counter disk rotation }\left(\mathrm{km} \mathrm{s}^{-1}\right)\end{array}$ \\
\hline 171 & err_v_phi[2] & Error of v_phi[2] \\
\hline 172 & $\mathrm{v} \_z[2]$ & $\begin{array}{l}\text { Galactic space velocities in the } \mathrm{z} \text { direction in a Galactocentric cylindrical polar coordinate system, } \\
\text { computed from dist_em and the corrected PPMXL and UCAC4 proper motions, respectively, } \\
\text { positive towards the North Galactic Pole }\left(\mathrm{km} \mathrm{s}^{-1}\right)\end{array}$ \\
\hline 173 & err_v_z[2] & Error of v_z[2] \\
\hline 174 & $\mathrm{e}[2]$ & Orbital eccentricities computed from dist_em and the corrected PPMXL and UCAC4 proper motions, respectively \\
\hline 175 & Rapo[2] & Maximum Galactic radii reached by the orbits computed with PPMXL and UCAC4 proper motions, respectively (kpc) \\
\hline 176 & Rperi[2] & Minimum Galactic radii reached by the orbits computed with PPMXL and UCAC4 proper motions, respectively (kpc) \\
\hline 177 & $\mathrm{zmin}[2]$ & Minimum height reached by the orbits computed with PPMXL and UCAC4 proper motions, respectively (kpc) \\
\hline 178 & $\operatorname{zmax}[2]$ & Maximum height reached by the orbits computed with PPMXL and UCAC4 proper motions, respectively (kpc) \\
\hline
\end{tabular}

